\title{
The Broad Absorption Line Tidal Disruption Event iPTF15af: Optical and Ultraviolet Evolution
}

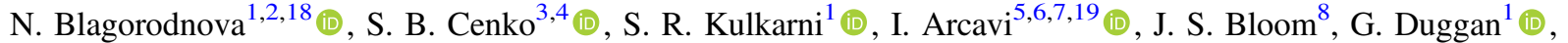

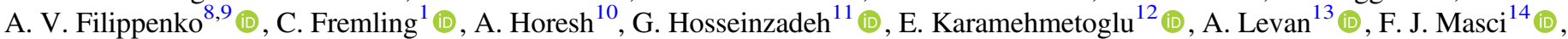 \\ P. E. Nugent ${ }^{8,15}$ (1) D. R. Pasham ${ }^{16,19}$, S. Veilleux ${ }^{3,17}$ (1) , R. Walters ${ }^{1}$, L. Yan ${ }^{1}$, and W. Zheng ${ }^{8}$ \\ ${ }^{1}$ Cahill Center for Astrophysics, California Institute of Technology, Pasadena, CA 91125, USA; N.Blagorodnova@astro.ru.nl \\ ${ }_{3}^{2}$ Department of Astrophysics/IMAPP, Radboud University, Nijmegen, The Netherlands \\ ${ }^{3}$ Joint Space-Science Institute, University of Maryland, College Park, MD 20742, USA \\ ${ }^{4}$ NASA Goddard Space Flight Center, Mail Code 661, Greenbelt, MD 20771, USA \\ ${ }^{5}$ Department of Physics, University of California, Santa Barbara, CA 93106-9530, USA \\ ${ }^{6}$ Las Cumbres Observatory, 6740 Cortona Drive, Suite 102, Goleta, CA 93117-5575, USA \\ ${ }^{7}$ The School of Physics and Astronomy, Tel Aviv University, Tel Aviv 69978, Israel \\ ${ }^{8}$ Department of Astronomy, University of California, Berkeley, CA 94720-3411, USA \\ ${ }^{9}$ Miller Senior Fellow, Miller Institute for Basic Research in Science, University of California, Berkeley, CA 94720, USA \\ ${ }^{10}$ Racah Institute of Physics, The Hebrew University of Jerusalem, Jerusalem, 91904, Israel \\ ${ }^{11}$ Harvard-Smithsonian Center for Astrophysics, 60 Garden Street, Cambridge, MA 02138-1516, USA \\ 12 Harvard-Smithsonian Center for Astrophysics, 60 Garden Street, Cambridge, MA 02138-1516, USA
Department of Astronomy, The Oskar Klein Centre, Stockholm University, AlbaNova, SE-10691, Stockholm, Sweden \\ ${ }^{13}$ Department of Physics, University of Warwick, Coventry, CV4 7AL, UK
${ }^{14}$ fnfrared Processing and Analysis Center, California Institute of Technology, Pasadena, CA 91125, USA \\ ${ }^{15}$ Computational Science Department, Lawrence Berkeley National Laboratory, 1 Cyclotron Road, MS 50B-4206, Berkeley, CA 94720, USA \\ ${ }^{16}$ Massachusetts Institute of Technology, Cambridge, MA 02139, USA \\ ${ }^{17}$ Department of Astronomy, University of Maryland, Stadium Drive, College Park, MD 20742-2421, USA \\ Received 2018 September 19; revised 2019 January 23; accepted 2019 February 1; published 2019 March 7
}

\begin{abstract}
We present multiwavelength observations of the tidal disruption event (TDE) iPTF15af, discovered by the intermediate Palomar Transient Factory survey at redshift $z=0.07897$. The optical and ultraviolet (UV) light curves of the transient show a slow decay over 5 months, in agreement with previous optically discovered TDEs. It also has a comparable blackbody peak luminosity of $L_{\text {peak }} \approx 1.5 \times 10^{44} \mathrm{erg} \mathrm{s}^{-1}$. The inferred temperature from the optical and UV data shows a value of $(3-5) \times 10^{4} \mathrm{~K}$. The transient is not detected in $\mathrm{X}$-rays up to $L_{\mathrm{X}}<3 \times 10^{42} \mathrm{erg} \mathrm{s}^{-1}$ within the first 5 months after discovery. The optical spectra exhibit two distinct broad emission lines in the He II region, and at later times also $\mathrm{H} \alpha$ emission. Additionally, emission from [N III] and [O III] is detected, likely produced by the Bowen fluorescence effect. UV spectra reveal broad emission and absorption lines associated with high-ionization states of N V, C IV, Si IV, and possibly P V. These features, analogous to those of broad absorption line quasars (BAL QSOs), require an absorber with column densities $N_{\mathrm{H}}>10^{23} \mathrm{~cm}^{-2}$. This optically thick gas would also explain the nondetection in soft X-rays. The profile of the absorption lines with the highest column density material at the largest velocity is opposite that of BAL QSOs. We suggest that radiation pressure generated by the TDE flare at early times could have provided the initial acceleration mechanism for this gas. Spectral UV line monitoring of future TDEs could test this proposal.
\end{abstract}

Key words: accretion, accretion disks - black hole physics - galaxies: nuclei - stars: individual (iPTF15af)

Supporting material: machine-readable table

\section{Introduction}

Most of the galaxies in our nearby universe contain a supermassive black hole (SMBH) at their cores (Kormendy \& Richstone 1995). Occasionally, accretion of gas in the vicinity of the SMBH reveals its presence as an active galactic nucleus (AGN). However, a large fraction of SMBHs appear to be dormant, as their activity is not directly revealed through observations in the electromagnetic spectrum. Sometimes the disruption of a star in the vicinity of the $\mathrm{SMBH}-\mathrm{a}$ tidal disruption event (TDE) — will produce a bright flare, detectable at multiple wavelengths. This new energy release episode will shed light on these otherwise quiescent SMBHs, allowing us to study in more detail their characteristics, such as their masses

\footnotetext{
18 VENI Fellow.

19 Einstein Fellow.
}

(Mockler et al. 2019) or spin distributions (Stone et al. 2019). In addition, the shocks, circularization, and accretion of the stellar debris onto the SMBH can reveal clues to better understand the more stable AGN activity or the unusual behavior of variable quasars (LaMassa et al. 2015).

According to theory, flares from TDEs were expected to originate in the innermost regions of the accretion disks (Rees 1988), which translate into soft X-ray emission and extreme-ultraviolet (EUV) emission. Consequently, searches for TDEs in archival data started in X-rays using ROSAT and the XMM-Newton Slew Survey (Bade et al. 1996; Komossa \& Bade 1999; Saxton et al. 2012). Subsequently, transient UV emission was also associated with activity produced by stellar disruptions (Gezari et al. 2006, 2008), and the search progressed at optical (van Velzen et al. 2011; Arcavi et al. 2014), gamma-ray, and radio wavelengths (Bloom et al. 2011; Burrows et al. 2011; Cenko et al. 2012). Currently, optical 
transient surveys are the most active discovery tool, with a total of $\sim 25$ optical TDE candidates reported to date. ${ }^{20}$

The majority of recent optical TDE discoveries were monitored in the UV using broadband photometry with the Neil Gehrels Swift Observatory (Swift; Gehrels et al. 2004). The sample has shown significantly higher temperature in the continuum than supernovae, with $T_{\text {eff }} \approx(20-80) \times 10^{3} \mathrm{~K}$ (see sample in Hung et al. 2017), suggesting that the bulk of the energy was released in the UV, inaccessible to ground-based telescopes. To date, rest-frame UV spectra of only five TDEs have been published, including the object discussed herein. While the optical spectra of these events are usually marked by He II, He I, and occasionally $\mathrm{H}$ broad emission lines (BELs; Holoien et al. 2016a; Blagorodnova et al. 2017), far-UV spectroscopy of ASASSN-14li (Cenko et al. 2016), iPTF16fnl (Brown et al. 2018), and iPTF15af (also published in Yang et al. 2017) have shown the presence of broad collisionally excited lines of Ly $\alpha, \mathrm{C}$ IV, N V, and Si IV. In contrast, lowionization lines of Fe II and $\mathrm{Mg}$ II dominated the UV spectra for PS1-11 af (Chornock et al. 2014) and PS16dtm (Blanchard et al. 2017). The observed diversity suggests that UV wavelengths are especially relevant for characterizing the geometry, density, and kinematics of the stellar debris and preexisting circumnuclear material.

Here we present the discovery and follow-up observations at optical, UV, mid-IR, X-ray, and radio wavelengths of iPTF15af, a TDE discovered by the intermediate Palomar Transient Factory (iPTF; Rau et al. 2009) survey at redshift $z=0.07897$. We show that the photometric and spectroscopic evolution of this event resembles other tidal disruption flares reported to date. The broad absorption component observed in the UV spectrum suggests the presence of high-velocity outflows, similar to the ones observed in broad absorption line quasars (BAL QSOs).

Section 2 describes the discovery, the host galaxy, and our multiwavelength follow-up observations. Sections 3 and 4 describe the photometric and spectroscopic analysis of the object, respectively. In Section 5 we compare the UV spectra of iPTF15af to those of other TDEs and QSOs. In Section 6 we summarize our conclusions.

In this work, we assume a flat cosmology and values of $H_{0}=70 \mathrm{~km} \mathrm{~s}^{-1} \mathrm{Mpc}^{-1}, \Omega_{M}=0.30$, and $\Omega_{\Lambda}=0.70$.

\section{Discovery and Observations}

iPTF15af was discovered by the iPTF survey on UTC 2015 January 15.3 (MJD 57,037.3). The object was detected in three of the five difference images taken on that night. The average AB magnitude (Oke \& Gunn 1983) measured by the pipeline (Masci et al. 2017) was $R=20.7 \pm 0.2$ in the iPTF Mould $R$ band. Stacked forced photometry allowed us to recover the transient in archival data at 25 days prior to its discovery in single-epoch images.

The central location of the transient in its host galaxy (see Figure 1) awarded the transient multiwavelength follow-up observations and a spectroscopic classification a week later. The spectrum confirmed the presence of strong He II lines, characteristic of an optical TDE candidate (Gezari et al. 2012; Arcavi et al. 2014).

From the averaged positions of the residuals in the $r$ band, we derive the location of the transient to be $\alpha=08^{\mathrm{h}} 48^{\mathrm{m}} 28^{\mathrm{s}} .12$,

\footnotetext{
${ }^{20}$ Based on reports to the TDE Open Catalogue https://tde.space.
}

$\delta=+22^{\circ} 03^{\prime} 33^{\prime \prime} 575$ (J2000), with a standard deviation in both coordinates of $1 \sigma=120$ mas. This position is offset from the central location of the galaxy core by 230 mas, which is consistent with the nucleus of the galaxy within $2 \sigma$. The Milky Way extinction along the line of sight is $A_{V}=0.093 \mathrm{mag}$ (Schlafly \& Finkbeiner 2011).

\subsection{Host Galaxy}

The transient's host galaxy SDSS J084828.13+220333.4 is located inside the footprint of the Sloan Digital Sky Survey (SDSS) DR12 (Alam et al. 2015). An archival spectrum of the host was obtained on MJD 53,379 as part of the SDSS spectroscopic survey. We do not identify major lines associated with star formation or AGNs. The spectroscopic redshift of the galaxy is $z=0.07897 \pm 0.00004$, which corresponds to a luminosity distance $D_{L}=358 \mathrm{Mpc}$ (distance modulus $\mu=$ $37.8 \mathrm{mag}$ ).

Archival photometry of the host is provided in Table 1. The limit for the GALEX far-ultraviolet (FUV) band was obtained from synthetic photometry of the best-fit galaxy model from the Bruzual \& Charlot (2003) spectral library. The spectrum was reddened and normalized to the SDSS $g$-band measurement.

Literature modeling analysis of the light profile of the galaxy shows that the values derived for its bulge-to-total light ratios are $(B / T)_{g}=0.50$ and $(B / T)_{r}=0.56$ in the $g$ and $r$ bands, respectively (Simard et al. 2011). The stellar mass for the host and its bulge, $\log \left(M_{*} / M_{\odot}\right)=10.360_{-0.138}^{+0.099}$ and $\log \left(M_{b} / M_{\odot}\right)=$ $9.99_{-0.15}^{+0.12}$, show that $\sim 40 \%$ of the mass is concentrated in the bulge of the galaxy (Mendel et al. 2014). Galaxies with enhanced stellar density cores are common among TDE hosts (Graur et al. 2018), showing unusually high $B / T$ values (LawSmith et al. 2017). High-resolution spectroscopy of the galaxy has allowed us to measure the velocity dispersion in the host, leading to an $M-\sigma$ estimate of $\log _{10}\left(M_{\mathrm{BH}} / M_{\odot}\right)=6.88_{-0.38}^{0.38}$ (Wevers et al. 2017).

The low specific star formation rate (sSFR) of the host, $\log _{10}(\mathrm{sSFR} / \mathrm{yr})=-14.87_{-0.10}^{+3.55}$ (Chang et al. 2015), shows that at the present time the galaxy is not actively forming stars. However, further analysis reveals that the host experienced a short ( $\sim 25 \mathrm{Myr}$ ) burst of star formation $\sim 600 \mathrm{Myr}$ ago (French et al. 2017). However, this burst could account for only a small fraction of the mass $(5 \%)$.

Since 2005, the host galaxy of the transient has been continuously monitored by the Catalina Real-time Transient Survey (Drake et al. 2009). During the past $\sim 10 \mathrm{yr}$, the host galaxy is well detected with an average magnitude of $V=17.67 \pm 0.11$, which appears constant within the errors. Nuclear activity is also unlikely, as inferred from the quiescent mid-IR Wide-field Infrared Survey Explorer (WISE; Wright et al. 2010) color $W 1-W 2=0.177$, away from the locus determined by $W 1-W 2 \geqslant 0.8$ (Stern et al. 2012). Based on the lack of AGN features in the spectrum, the lack of variability, and no mid-IR excess, we can rule out previous AGN activity in the host at least $\sim 10 \mathrm{yr}$ before the discovery of the source.

\subsection{Ground-based Photometry}

After its discovery, the transient was simultaneously monitored for 3 months in the $R$ band with the Palomar 48-inch telescope (P48) and in the gri bands with the Palomar 60 -inch telescope (P60). The P48 data reduction is described by Laher et al. (2014), while the photometric calibration and the 

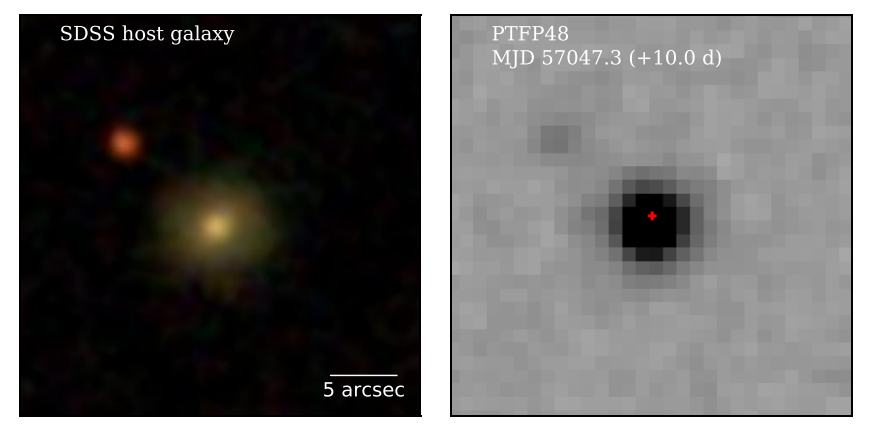

Figure 1. Left: archival SDSS color composite of the host galaxy. Right: iPTF P48 image with the transient at 10 days after discovery (around peak magnitude). The red plus sign marks the location of the transient.

Table 1

Photometry of SDSS J084828.13+220333.4

\begin{tabular}{|c|c|c|c|}
\hline Survey & Band & $\begin{array}{l}\text { Magnitude } \\
\text { (mag) }\end{array}$ & References \\
\hline GALEX & $\mathrm{FUV}_{\mathrm{AB}}$ & $>24.6^{\mathrm{a}}$ & (1) \\
\hline GALEX & $\mathrm{NUV}_{\mathrm{AB}}$ & $24.02 \pm 0.48^{\mathrm{b}}$ & (1) \\
\hline SDSS DR12 & $u$ & $20.286 \pm 0.084^{\mathrm{c}}$ & (2) \\
\hline SDSS DR12 & $g$ & $18.520 \pm 0.010^{c}$ & (2) \\
\hline SDSS DR12 & $r$ & $17.606 \pm 0.007^{c}$ & (2) \\
\hline SDSS DR12 & $i$ & $17.186 \pm 0.008^{c}$ & (2) \\
\hline SDSS DR12 & $z$ & $16.883 \pm 0.019^{c}$ & (2) \\
\hline 2MASS & $J$ & $16.433 \pm 0.132^{\mathrm{d}}$ & (3) \\
\hline 2MASS & $H$ & $15.671 \pm 0.148^{d}$ & (3) \\
\hline 2MASS & $K$ & $15.547 \pm 0.175^{\mathrm{d}}$ & (3) \\
\hline WISE & $W 1$ & $14.809 \pm 0.033^{\mathrm{d}}$ & (4) \\
\hline WISE & $W 2$ & $14.632 \pm 0.068^{\mathrm{d}}$ & (4) \\
\hline WISE & $W 3$ & $>12.309^{\mathrm{d}}$ & (4) \\
\hline WISE & $W 4$ & $>8.681^{\mathrm{d}}$ & (4) \\
\hline NVSS & $1.4 \mathrm{GHz}$ & $<1.8 \mathrm{mJy}$ & (5) \\
\hline$R O S A T$ & $0.1-2.4 \mathrm{keV}$ & $<3.9 \times 10^{-13} \mathrm{erg} \mathrm{s}^{-1}$ & (6) \\
\hline
\end{tabular}

Notes.

a Synthetic photometry.

b Measured within 7!' 5 diameter aperture.

c Model magnitude.

d PSF magnitude.

References. (1) Bianchi et al. (2011); (2) Alam et al. (2015); (3) Skrutskie et al. (2006); (4) Wright et al. (2010); (5) Condon et al. (1998); (6) Voges et al. (1999).

system are discussed by Ofek et al. (2012). The differenceimage photometric measurements were obtained with PTFIDE (Masci et al. 2017) and FPipe (Fremling et al. 2016) for P48 and P60, respectively. The zero-points were calibrated using the SDSS DR13 catalog (SDSS Collaboration et al. 2017). Simultaneously, we also obtained follow-up photometry in the BVgri bands with Las Cumbres Observatory's $1 \mathrm{~m}$ telescope in Texas (Brown et al. 2013). The point-spread function photometry was obtained using lcogtsnpipe (Valenti et al. 2016), which is calibrated to the APASS 9 (Henden et al. 2016) and SDSS DR13 catalogs for $B V$ and gri, respectively. Deeper, prediscovery photometric measurements were obtained by combining the flux from all available nightly sets of five images. The photometric evolution of iPTF15af is shown in Figure 2, and the difference-imaging measurements are available as part of Table 2 .

The first noticeable characteristic of this event is its slow evolution in the UV and optical bands. Since our first detections in $r$, there is a slow brightening by $\sim 1$ mag on a timescale of 30 days. After that, the optical bands reach a plateau phase, lasting $\sim 3$ months. The observed apparent magnitudes at peak (around MJD 57,370) are $m_{g}=19.8 \pm$ $0.1 \mathrm{mag}$ and $m_{r}=20.2 \pm 0.1 \mathrm{mag}$, corresponding to absolute magnitudes (corrected for foreground extinction) of $M_{g}=$ $-17.9 \pm 0.1 \mathrm{mag}$ and $M_{r}=-17.7 \pm 0.1 \mathrm{mag}$. Because of the faint apparent magnitude of the object, our follow-up observations are nondetections in the optical bands after the fourth month; apparently, by that time the transient had faded below $\sim 21$ mag in $g$ and 22 in $r$.

\subsection{Swift Photometry}

Following the optical discovery of iPTF15af, we initiated follow-up observations with the Ultra-Violet Optical Telescope (UVOT; Roming et al. 2005) on board the Swift satellite. The data were obtained in three successive campaigns in all six UVOT filters. We used the software package UVOTSOURCE to obtain the counts of the source within a $5^{\prime \prime}$ aperture radius. The background was computed from an off-target sky region using an aperture of $12^{\prime \prime}$. The magnitudes were derived using the latest UVOT calibration (Poole et al. 2008; Breeveld et al. 2010). The aperture magnitude measurements are listed in Table 2.

The evolution of the Swift photometry is shown in Figure 2. While the reddest bands, with higher contamination from host galaxy light, follow the plateau observed in the optical bands, the UV bands $U V W 2$ and $U V M 2$ show a declining trend after 50 days, which places an approximate date of the peak at $\sim 40$ days after discovery in the rest frame of the source (around MJD 57,077.3). We note that while at later times the UV emission has considerably faded, our measurements are still 4 mag brighter than the archival ones reported by $G A L E X$. The flattening of UV emission at late times is consistent with the trend observed for a sample of optical TDEs, observed 5-10 yr after the flare (van Velzen et al. 2018).

\subsection{Optical Spectroscopy}

Optical spectra were obtained with the Low Resolution Imaging Spectrometer (Oke et al. 1995) on the Keck I $10 \mathrm{~m}$ telescope and were reduced using the automated reduction pipeline in IDL 1 pipe, ${ }^{21}$ developed by D. Perley.

The full spectral $\log$ is provided in Table 3. Upon the publication of the article, all spectra will be made publicly available via the WISeREP repository (Yaron \& Gal-Yam 2012).

The spectral evolution of the event from +7 to +149 days after discovery is shown in the left panel of Figure 3. In order to optimize the host galaxy subtraction, we used the same configuration to obtain a late-time spectrum of the host at +1035 days after discovery, when the TDE contribution was completely gone. Visual inspection of our spectrum at +149 days after discovery confirms that for that epoch there was still significant residual flux from the transient.

\subsection{UV Spectroscopy}

UV spectroscopy of iPTF15af was obtained (PI S. B. Cenko, proposal GO-13853) with the Space Telescope Imaging

\footnotetext{
$\overline{{ }^{21} \text { http://www.astro.caltech.edu/ dperley/programs/lpipe.html }}$
} 


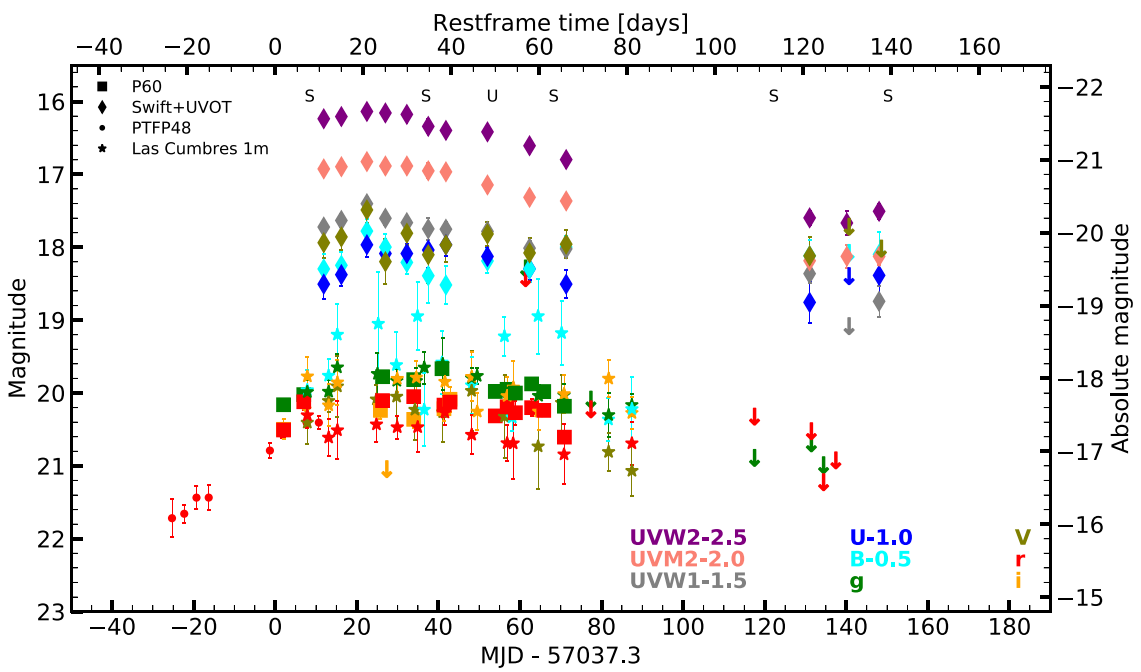

Figure 2. iPTF15af photometric measurements, corrected for Galactic extinction. For display purposes, the data have been binned with a bin size of 3 days. PTF measurements are obtained from difference photometry. The Swift measurements correspond to aperture photometry. Arrows show the upper limits. The epochs for optical spectra are marked with "S" and the epoch of the UV spectrum with "U." We use the discovery date as our reference epoch.

Table 2

Optical Difference Imaging and Swift UV Aperture Photometry of iPTF15af in the AB Magnitude System ${ }^{\text {a }}$

\begin{tabular}{|c|c|c|c|c|c|c|c|c|c|c|c|}
\hline $\begin{array}{l}\text { MJD } \\
\text { (days) }\end{array}$ & $\begin{array}{l}\text { Phase }^{\mathrm{b}} \\
\text { (days) }\end{array}$ & $\begin{array}{c}\text { Telescope } \\
+ \text { Instrument }\end{array}$ & $\begin{array}{l}U V W 1 \\
(\mathrm{mag})\end{array}$ & $\begin{array}{l}U V M 2 \\
(\mathrm{mag})\end{array}$ & $\begin{array}{l}U V W 2 \\
(\mathrm{mag})\end{array}$ & $\begin{array}{c}U \\
(\mathrm{mag})\end{array}$ & $\begin{array}{c}B \\
(\mathrm{mag})\end{array}$ & $\begin{array}{c}V \\
(\mathrm{mag})\end{array}$ & $\begin{array}{c}g \\
(\mathrm{mag})\end{array}$ & $\begin{array}{c}r \\
(\mathrm{mag})\end{array}$ & $\begin{array}{c}i \\
(\mathrm{mag})\end{array}$ \\
\hline $56,664.0$ & -373.3 & PTFP48 & $\ldots$ & $\ldots$ & $\ldots$ & $\ldots$ & $\ldots$ & $\ldots$ & $\ldots$ & $>23.29$ & $\ldots$ \\
\hline $56,667.0$ & -370.3 & PTFP48 & $\ldots$ & $\ldots$ & $\ldots$ & $\ldots$ & $\ldots$ & $\ldots$ & $\ldots$ & $>22.20$ & $\ldots$ \\
\hline $56,670.0$ & -367.3 & PTFP48 & $\ldots$ & $\ldots$ & $\ldots$ & $\ldots$ & $\ldots$ & $\ldots$ & $\ldots$ & $>20.47$ & $\ldots$ \\
\hline $56,769.0$ & -268.3 & PTFP48 & $\ldots$ & $\ldots$ & $\ldots$ & $\ldots$ & $\ldots$ & $\ldots$ & $\ldots$ & $>21.89$ & $\ldots$ \\
\hline $57,012.0$ & -25.3 & PTFP48 & $\ldots$ & $\ldots$ & $\ldots$ & $\ldots$ & $\ldots$ & $\ldots$ & $\ldots$ & $21.79 \pm 0.26$ & $\ldots$ \\
\hline $57,015.0$ & -22.3 & PTFP48 & $\ldots$ & $\ldots$ & $\ldots$ & $\ldots$ & $\ldots$ & $\ldots$ & $\ldots$ & $21.73 \pm 0.12$ & $\ldots$ \\
\hline $57,018.0$ & -19.3 & PTFP48 & $\ldots$ & $\ldots$ & $\ldots$ & $\ldots$ & $\ldots$ & $\ldots$ & $\ldots$ & $21.51 \pm 0.16$ & $\ldots$ \\
\hline $57,021.0$ & -16.3 & PTFP48 & $\ldots$ & $\ldots$ & $\ldots$ & $\ldots$ & $\ldots$ & $\ldots$ & $\ldots$ & $21.51 \pm 0.18$ & $\ldots$ \\
\hline $57,036.0$ & -1.3 & PTFP48 & $\ldots$ & $\ldots$ & $\ldots$ & $\ldots$ & $\ldots$ & $\ldots$ & $\ldots$ & $20.87 \pm 0.11$ & $\ldots$ \\
\hline $57,039.3$ & 2.0 & P60+SEDM & $\ldots$ & $\ldots$ & $\ldots$ & $\ldots$ & $\ldots$ & $\ldots$ & $20.27 \pm 0.06$ & $20.58 \pm 0.10$ & $20.55 \pm 0.14$ \\
\hline $57,039.0$ & 1.7 & PTFP48 & $\ldots$ & $\ldots$ & $\ldots$ & $\ldots$ & $\ldots$ & $\ldots$ & $\ldots$ & $20.64 \pm 0.05$ & $\ldots$ \\
\hline $57,044.3$ & 7.0 & P60+SEDM & $\ldots$ & $\ldots$ & $\ldots$ & $\ldots$ & $\ldots$ & $\ldots$ & $20.13 \pm 0.07$ & $20.20 \pm 0.12$ & $>20.20$ \\
\hline$\ldots$ & $\ldots$ & $\ldots$ & $\ldots$ & $\ldots$ & $\ldots$ & $\ldots$ & $\ldots$ & $\ldots$ & $\ldots$ & $\ldots$ & \\
\hline
\end{tabular}

Notes.

a The $r$-band column for P48 contains measurements in the Mould $R$ filter system. These data are not corrected for Galactic extinction.

b The phase is with respect to the date of discovery MJD 57,037.3.

(This table is available in its entirety in machine-readable form.)

Table 3

Log of Ground-based Spectroscopic Observations of iPTF15af ${ }^{a}$

\begin{tabular}{|c|c|c|c|c|c|}
\hline $\begin{array}{l}\text { Phase }^{\mathrm{b}} \\
\text { (days) }\end{array}$ & $\begin{array}{l}\text { MJD } \\
\text { (days) }\end{array}$ & UTC & $\begin{array}{l}\text { Telescope } \\
+ \text { Instrument }\end{array}$ & $\begin{array}{l}\text { P.A. } \\
\text { (deg) }\end{array}$ & $\begin{array}{c}\text { Exposure } \\
\text { (s) }\end{array}$ \\
\hline+7 & $57,044.5$ & 2015 Jan 22 11:38:56 & Keck I+LRIS & 105 & 1240 \\
\hline+36 & $57,073.5$ & 2015 Feb 20 12:59:35 & Keck I+LRIS & 268 & 600 \\
\hline+67 & $57,104.3$ & 2015 Mar 23 07:16:37 & Keck I+LRIS & 230 & 820 \\
\hline+121 & $57,158.3$ & 2015 May 16 06:17:20 & Keck I+LRIS & 87 & 1200 \\
\hline+149 & $57,186.3$ & 2015 Jun 13 06:11:08 & Keck I+LRIS & 270 & 610 \\
\hline+1035 & $58,072.6$ & 2017 Nov 15 13:33:51 & Keck I+LRIS & 272 & 1160 \\
\hline
\end{tabular}

Notes.

a All observations were conducted with a combination of grism 400/3400 and grating 400/8500, and a long $1^{\prime \prime}$-wide slit, providing a resolution of $\sim 7 \AA$.

$\mathrm{b}$ The phase is relative to discovery date with MJD 57,037.3.

Spectrometer on the Hubble Space Telescope (HST) on UTC 2015 March 08.1 (MJD 57,089.1), at 52 days after discovery. The combined data from the FUV and the near-UV (NUV) MAMA detectors provide coverage in the 1145-3145 $\AA$ range.
The spectrum is represented in Figure 4, and the log of the UV observations is provided in Table 4.

Given the good signal-to-noise ratio of the trace in the twodimensional images, we used the one-dimensional spectra output 


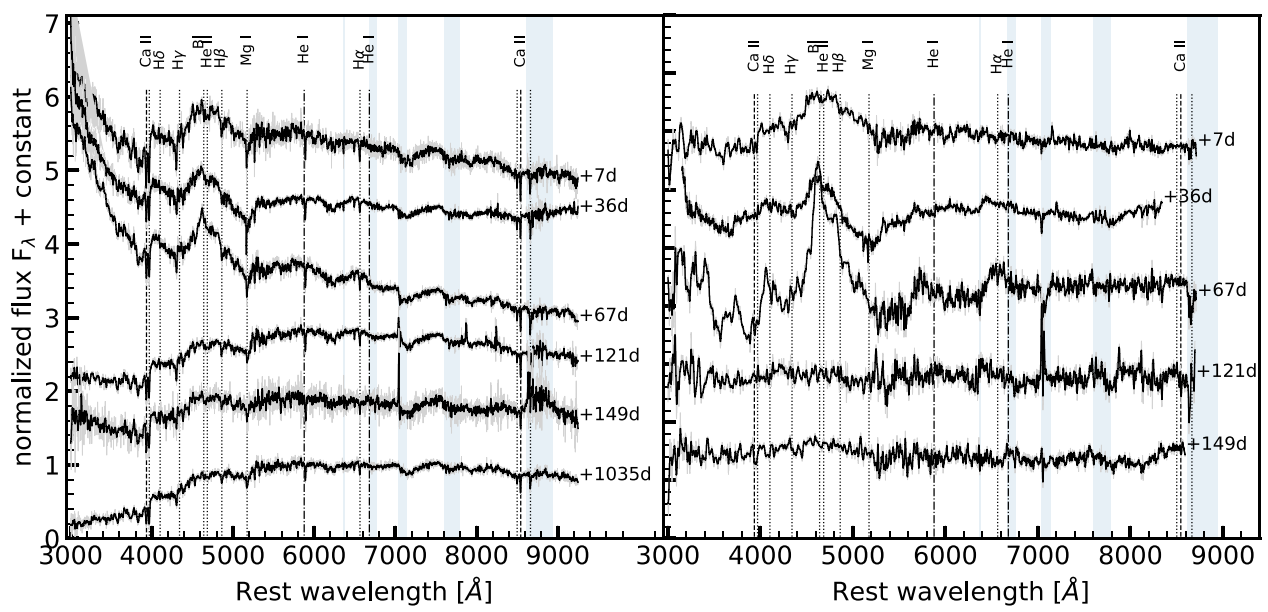

Figure 3. Left: rest-frame optical spectra of iPTF15af obtained with LRIS on the Keck I telescope. The spectra are not corrected for Milky Way extinction. Right: subtracted residuals for each epoch using the spectrum of the host at +1035 days plus a blackbody. The broad absorption feature around $5200 \AA$ noticeable at +36 days is related to the dichroic, which was placed at rest frame $5600 \AA$. The most relevant element transition lines are marked.

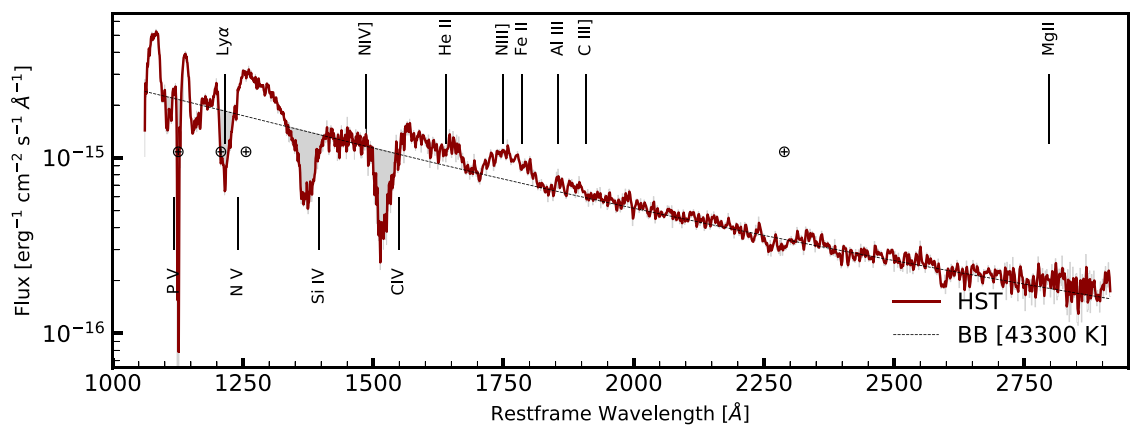

Figure 4. HST/STIS spectrum of iPTF15af at 52 days after discovery (red dark line) and the best-fit blackbody emission to the segment between 2000 and $3000 \AA$ (black dashed line). The major broad emission lines have been marked in the upper part of the plot, and the major BALs are shown with shaded areas in the bottom, along with a label. The spectrum has been corrected for Galactic extinction. The regions of geocoronal airglow lines are marked with $\oplus$ symbols.

Table 4

Log of UV Spectroscopy of iPTF15af with $H S T^{\mathrm{a}}$

\begin{tabular}{llccc}
\hline \hline UTC & Grating & $\begin{array}{c}\text { Scale } \\
\left(\AA^{-1} \text { pixel }^{-1}\right)\end{array}$ & $\begin{array}{c}\text { Res. Power } \\
(\lambda / \Delta \lambda)\end{array}$ & $\begin{array}{c}\text { Exposure } \\
(\mathrm{s})\end{array}$ \\
\hline 2015 Mar 8 01:12:13 & G230L & 1.58 & $500-1010$ & 2130.0 \\
2015 Mar 8 02:32:38 & G230L & 1.58 & $500-1010$ & 2922.0 \\
2015 Mar 8 04:08:06 & G140L & 0.60 & $960-1440$ & 2922.0 \\
2015 Mar 8 05:43:35 & G140L & 0.60 & $960-1440$ & 2922.0 \\
2015 Mar 8 07:19:03 & G140L & 0.60 & $960-1440$ & 2922.0 \\
\hline
\end{tabular}

Note.

${ }^{a}$ STIS instrument and FUV-MAMA and NUV-MAMA detectors. All measurements were taken with slit $52 \times 0.2$.

by the $H S T$ pipeline. The spectra from each detector were combined using an inverse-variance-weighted average of the onedimensional spectra.

\subsection{X-Rays}

We used the X-ray Telescope (XRT; Burrows et al. 2005) on board the Swift satellite to observe iPTF15af in the $0.3-10.0 \mathrm{keV}$ bandpass using the photon counting mode. The observations, with typical exposure times of $\sim 2 \mathrm{ks}$, were taken simultaneously with the UVOT exposures. The single-epoch data only yielded nondetections, with an average $3 \sigma$ upper limit of $(5 \pm 2) \times 10^{-3}$ counts $^{-1}$ in this bandpass.
We measured the flux assuming that the source spectrum follows a power law with a photon index of $\Gamma=2$ and a lineof-sight absorption in the Milky Way of $N_{\mathrm{H}}=3 \times 10^{20} \mathrm{~cm}^{-2}$ (Willingale et al. 2013). Our count rate corresponds to an upper limit for the unabsorbed flux of $f=1.9_{-0.8}^{+0.5} \times$ $10^{-13} \mathrm{erg} \mathrm{cm}^{-2} \mathrm{~s}^{-1}$ in the $0.3-10.0 \mathrm{keV}$ bandpass. At the distance of iPTF15af, this value translates to an upper limit on the X-ray luminosity of $L_{\mathrm{X}}<3.0 \times 10^{42} \mathrm{erg} \mathrm{s}^{-1}$.

The total flux from the source in the stacked image of $33 \mathrm{ks}$ was estimated using the $99.7 \%$ Bayesian confidence interval (Kraft et al. 1991), encoded in the astropy Python package (assuming only a non-negativity prior). We used the exposureweighted correction factor provided by the Swift XRT pipeline to calculate the total of number of counts. The $3 \sigma$ level is $[0,3.6] \times 10^{-4}$ counts $\mathrm{s}^{-1}$, corresponding to an upper limit for the luminosity of $L_{\mathrm{X}} \leqslant 2.2 \times 10^{41} \mathrm{erg} \mathrm{s}^{-1}$ in the Swift band.

When comparing the X-ray luminosity of iPTF15af to that of other BAL QSOs, we first used the UV spectrum to derive the $\mathrm{UV} / \mathrm{X}$-ray luminosity relation, quantified by the $\alpha_{\mathrm{OX}}$ parameter (Avni \& Tananbaum 1982; Just et al. 2007),

$$
\alpha_{\mathrm{OX}} \equiv 0.3838 \log \left(\frac{f_{\nu}(2 \mathrm{keV})}{f_{\nu}(2500 \AA)}\right),
$$

where $f_{\nu}(2 \mathrm{keV})$ is the monochromatic unabsorbed flux density at $2 \mathrm{keV}$, derived using the stacked image counts and a power 


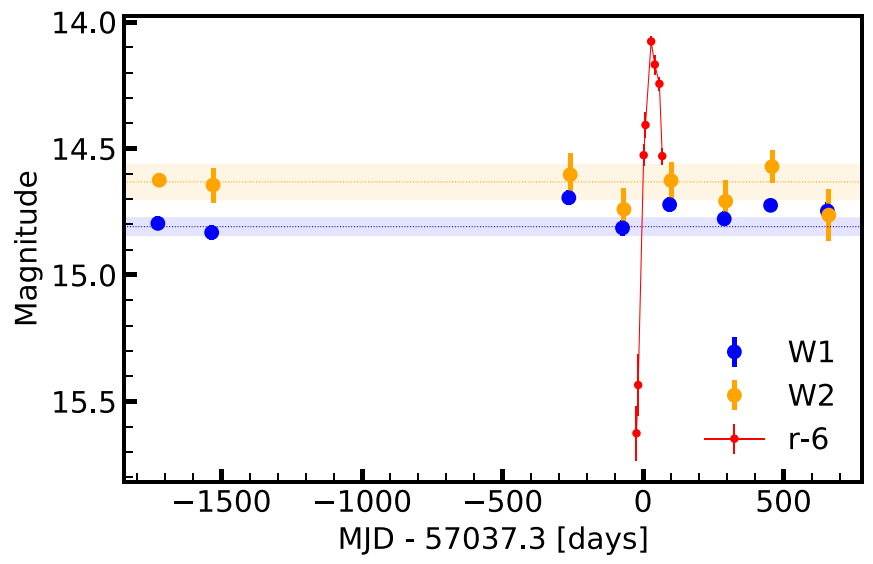

Figure 5. WISE (first two epochs) and NEOWISE (last six epochs) average weighted photometry is shown with orange and blue circles, respectively. The dotted line and shaded areas show the co-added magnitude and uncertainties, respectively, for the quiescent host from the AllWISE source catalog. The $r$-band binned photometry for the optical emission is shown with small red circles. An offset of -6 mag is applied.

law with photon index $\Gamma=2$, and $f_{\nu}(2500 \AA)$ is the flux density at $2500 \AA$, both in erg s $\mathrm{cm}^{-1} \mathrm{~Hz}^{-1}$.

For iPTF15af we obtained $\alpha_{\mathrm{OX}}=-1.8 \pm 0.1$ for the $\mathrm{X}$-ray upper confidence level interval. In order to compare this value to that of standard QSOs, we derived the expected X-ray luminosity for a typical QSO given its UV luminosity using the relation found by Just et al. (2007):

$$
\begin{aligned}
\alpha_{\mathrm{OX}}\left(L_{2500}\right)= & (-0.140 \pm 0.007) \log \left(L_{2500}\right) \\
& +(2.705 \pm 0.212) .
\end{aligned}
$$

The difference between the two ratios, parameterized as

$$
\Delta \alpha_{\mathrm{OX}} \equiv \alpha_{\mathrm{OX}}-\alpha_{\mathrm{OX}}\left(L_{2500}\right)
$$

is used as an indication of X-ray weakness as compared to the general QSO population.

For our TDE, we derive a value of $\Delta \alpha_{\mathrm{OX}}=-0.63$, which indicates that the transient is weaker in X-rays by a factor of $\sim 43$ than a typical QSO of the same UV luminosity. This value is in agreement with the weakest X-ray BAL QSOs from Gibson et al. (2009, their Figure 16).

\subsection{Radio}

Radio follow-up observations of iPTF15af were carried out with the Jansky Very Large Array (PI A. Horesh) on UTC 2015 January 31 , at 15 days after discovery. The source was not detected in either the $C(6.1 \mathrm{GHz})$, or $K(22 \mathrm{GHz})$ bands with an rms of 28 and $36 \mu \mathrm{Jy}$, respectively. The corresponding upper limits for the monochromatic radio luminosities are $\nu L_{\nu}<$ $2.6 \times 10^{37} \mathrm{erg} \mathrm{s}^{-1}$ and $\nu L_{\nu}<1.2 \times 10^{38} \mathrm{erg} \mathrm{s}^{-1}$ at 6.1 and $22 \mathrm{GHz}$

These early-time observations of iPTF15af cannot completely rule out an emission mechanism equivalent to that of ASASSN14li (Holoien et al. 2016a), which a month after discovery registered peak luminosities of $\nu L_{\nu} \approx 9 \times 10^{37} \mathrm{erg} \mathrm{s}^{-1}$ and $\nu L_{\nu} \approx 3 \times 10^{38} \mathrm{erg} \mathrm{s}^{-1}$ at 5 and $15.7 \mathrm{GHz}$, respectively (Alexander et al. 2016; van Velzen et al. 2016a). In case of jetted on-axis emission, these limits would constrain the jet energy to $E_{j}<10^{49} \mathrm{erg} \mathrm{s}^{-1}$ (Generozov et al. 2017).

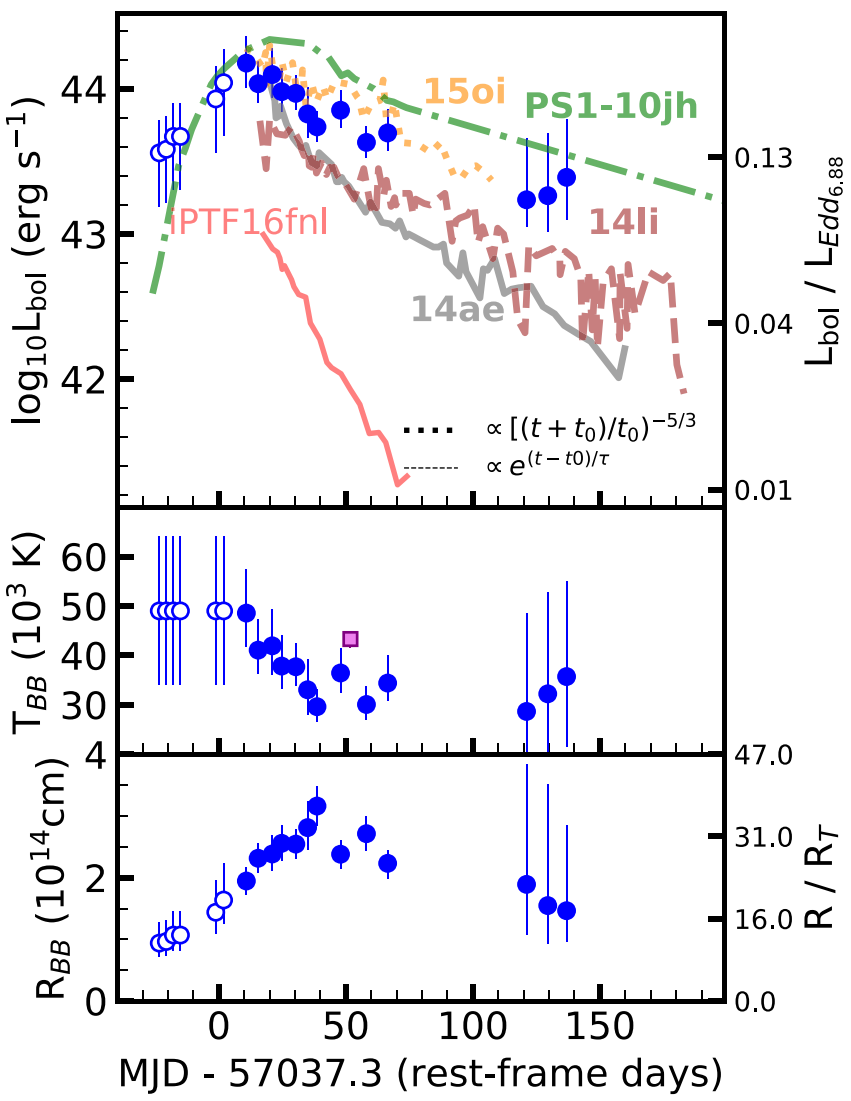

Figure 6. Top: bolometric blackbody light curve of iPTF15af. Blue filled circles represent the fits using extinction-corrected Swift bands. Open blue circles represent the values estimated using the $r$-band scaling. The purple square shows the temperature derived using the featureless portion of the HST UV spectrum. The right-hand top axis shows the bolometric luminosity as a fraction of the total Eddington luminosity for a $10^{6.88} M_{\odot}$ SMBH. Middle: temperature evolution. Bottom: evolution of the blackbody radius. The righthand axis shows the radius as a fraction of the tidal disruption radius for a $10^{6.88} M_{\odot}$ SMBH and a $1 M_{\odot}$ star. In all three plots the epoch of discovery has been adopted as the reference MJD.

\subsection{Mid-IR Photometry}

We retrieved archival mid-IR data prior to and after the event from the WISE survey and the 2017 release of NEOWISE (Mainzer et al. 2014). The WISE data correspond to the all-sky survey run in 3.4, 4.6, 12, and $22 \mu \mathrm{m}(W 1, W 2, W 3, W 4)$ taken in 2010. The NEOWISE data cover 3 years after the reactivation of the mission in 2013 December in the W1 and W2 bands.

Using the IRSA archive, ${ }^{22}$ we selected $W 1$ and $W 2$ magnitudes for sources detected in single exposures within $2^{\prime \prime}$ of the position of the transient. After discarding the measurements marked as upper limits ("U" value in the ph_qual column), we obtained the flux-weighted average combining the remaining $\sim 10$ measurements per epoch. The results are shown in Figure 5, where we also include the $r$-band light curve of the transient for comparison.

Although the NEOWISE light curve shows small variations around the archival value of the source in the allWISE catalog, none of them are more significant than $3 \sigma$. Hence, for iPTF15af we can rule out a detection of a strong IR echo as in the case of

22 http://irsa.ipac.caltech.edu 
PTF09ge (van Velzen et al. 2016b) or PS16dtm (Jiang et al. 2017).

\section{Spectral Energy Distribution (SED) Analysis}

For our SED analysis, we used Swift UVOT aperture photometry for $U V W 1, U V M 2$, and $U V W 2$ (subtracting the host synthetic magnitude from our best-fit model spectra) and difference-image photometry in the $r$ and $g$ bands, to derive the blackbody temperature and radius of the emission from the transient. We fixed the time of the Swift observations as our reference epoch and interpolated the optical bands to derive the magnitudes at the same epoch. The fluxes, corrected for Milky Way extinction, were fit with an SED corresponding to a single-temperature blackbody model. We have excluded the optical Swift bands from our analysis, as they had considerable contamination from the host galaxy, which was still present in our last epochs. The $i$ band from the P60 exhibited larger scatter than usual, so it was excluded as well.

The initial value for the temperature prior was derived from the fit to the UV spectrum (see Section 4.2). Our posterior distributions for temperature and radius are based on Markov Chain Monte Carlo simulations, run using the Python package emcee (Foreman-Mackey et al. 2013). The blackbody bolometric luminosity, along with the best fit for the temperature and the radius (median, $16 \%$ and $84 \%$ percentiles) as derived from the SED, is shown as filled circles in Figure 6 and given in Table 5.

The rising part in our light curve only contains optical measurements, mainly in $R$ band. Our Swift measurements start a month after the detection of the source in stacked iPTF data. In order to derive the luminosity and the radius of the emitting region without Swift NUV photometry, we proceed as follows. We initially assume that the temperature of the emission does not deviate significantly from our first estimate from Swift photometry. We use this value of $T_{\mathrm{BB}} \approx 49,000 \mathrm{~K}$ to simulate blackbody emission and scale it to our $r$-band measurements. The results are shown in Figure 6 as open circles. Their error bars indicate the change in luminosity and radius if our assumed temperature varied by $\pm 15,000 \mathrm{~K}$.

The bolometric light curve obtained for iPTF15af shows a slow rise over the initial 30 days, reaching $L_{\text {peak }}=1.5_{-0.5}^{+0.8} \times$ $10^{44} \mathrm{erg} \mathrm{s}^{-1}$ and gradually decaying over the next 5 months, providing a rest-frame $e$-folding time of $\tau \approx 68$ days. The overall luminosity and timescales for this TDE, as shown in Figure 6, agree well with those of PS1-10jh (Gezari et al. 2012) and slowly evolving events like ASASSN-15oi (Holoien et al. 2016b).

The integrated bolometric light curve over the observed interval (Figure 6) shows a total energy of $L \geqslant(8.0 \pm 1.1) \times$ $10^{50} \mathrm{erg}$. Assuming an efficiency of $\epsilon=0.1$ for transforming the accreted mass into the observed luminosity, $E_{\mathrm{rad}}=\epsilon M_{\mathrm{acc}} c^{2}$, the inferred lower limit for the mass of the accreted material is $M_{\text {acc }}=(4.5 \pm 0.6) \times 10^{-3} M_{\odot}$.

Analogous to the trend seen in other optical TDEs (see Figure 11 in Hung et al. 2017), the temperature of iPTF15af remains relatively constant for nearly 200 days. The emission initially appears hotter, with $T_{\mathrm{BB}} \approx 49,000 \mathrm{~K}$, and only 2 months later evolves toward lower temperatures in the $30,000 \mathrm{~K}$ range. The cooling is related to an expansion of the photosphere from

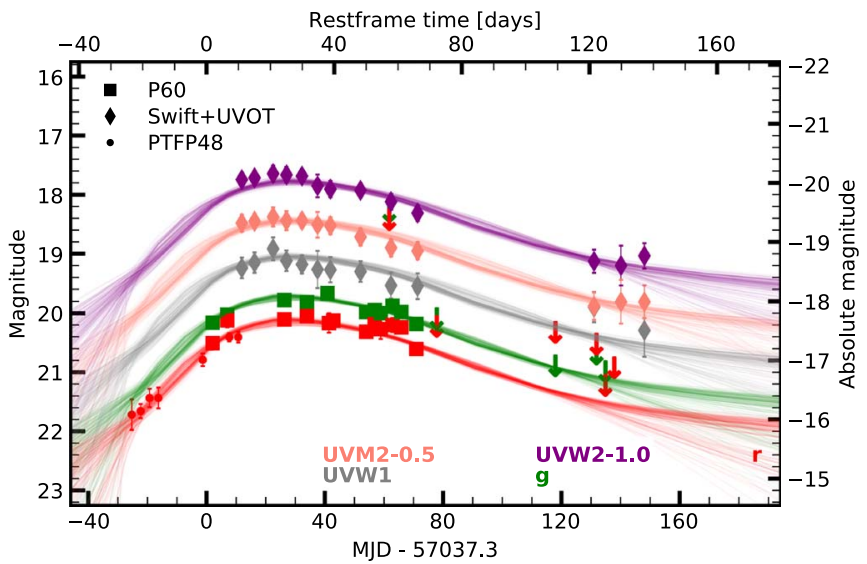

Figure 7. Markers correspond to iPTF15af optical and UV observations used for the modeling. The lines correspond to a set of best-fit models, obtained with MOSFiT. The color-coding for the observations and the models is the same.

Table 5

Best Blackbody Fits to the iPTF15af SED ${ }^{\mathrm{a}}$

\begin{tabular}{|c|c|c|c|c|}
\hline $\begin{array}{l}\text { MJD } \\
\text { (days) }\end{array}$ & $\begin{array}{l}\text { Rest Epoch } \\
\text { (days) }\end{array}$ & $\begin{array}{c}T_{\mathrm{BB}} \\
\left(10^{4} \mathrm{~K}\right)\end{array}$ & $\begin{array}{c}R_{\mathrm{BB}} \\
\left(10^{14} \mathrm{~cm}\right)\end{array}$ & $\begin{array}{c}L_{\mathrm{BB}} \\
\left(10^{43} \mathrm{erg} \mathrm{s}^{-1}\right)\end{array}$ \\
\hline $57,012^{*}$ & -23.4 & $4.90_{-1.50}^{+1.50}$ & $0.94_{-0.22}^{+0.34}$ & $3.62_{-2.07}^{+2.48}$ \\
\hline $57,015^{*}$ & -20.7 & $4.90_{-1.50}^{+1.50}$ & $0.97_{-0.23}^{+0.35}$ & $3.83_{-2.19}^{+2.62}$ \\
\hline $57,018^{*}$ & -17.9 & $4.90_{-1.50}^{+1.50}$ & $1.07_{-0.26}^{+0.39}$ & $4.70_{-2.68}^{+3.21}$ \\
\hline $57,021^{*}$ & -15.1 & $4.90_{-1.50}^{+1.50}$ & $1.07_{-0.26}^{+0.39}$ & $4.70_{-2.68}^{+3.22}$ \\
\hline $57,036^{*}$ & -1.2 & $4.90_{-1.50}^{+1.50}$ & $1.44_{-0.34}^{+0.52}$ & $8.51_{-4.86}^{+5.83}$ \\
\hline $57,039^{*}$ & 1.9 & $4.90_{-1.50}^{+1.50}$ & $1.64_{-0.39}^{+0.59}$ & $11.02_{-6.29}^{+7.54}$ \\
\hline 57,049 & 10.8 & $4.86_{-0.68}^{+0.88}$ & $1.95_{-0.22}^{+0.23}$ & $15.03_{-4.81}^{+8.14}$ \\
\hline 57,054 & 15.5 & $4.11_{-0.49}^{+0.62}$ & $2.32_{-0.23}^{+0.24}$ & $10.84_{-2.91}^{+4.58}$ \\
\hline 57,060 & 21.0 & $4.19_{-0.58}^{+0.74}$ & $2.38_{-0.27}^{+0.30}$ & $12.54_{-3.90}^{+6.48}$ \\
\hline 57,064 & 24.7 & $3.78_{-0.47}^{+0.62}$ & $2.56_{-0.29}^{+0.29}$ & $9.56_{-2.64}^{+4.34}$ \\
\hline 57,070 & 30.3 & $3.77_{-0.39}^{+0.47}$ & $2.55_{-0.23}^{+0.24}$ & $9.30_{-2.17}^{+3.09}$ \\
\hline 57,075 & 34.9 & $3.30_{-0.50}^{+0.62}$ & $2.81_{-0.36}^{+0.42}$ & $6.71_{-2.14}^{+3.49}$ \\
\hline 57,079 & 38.6 & $2.96_{-0.29}^{+0.36}$ & $3.16_{-0.32}^{+0.33}$ & $5.47_{-1.11}^{+1.58}$ \\
\hline 57,089 & 47.9 & $3.64_{-0.40}^{+0.50}$ & $2.38_{-0.23}^{+0.24}$ & $7.12_{-1.72}^{+2.65}$ \\
\hline 57,100 & 58.1 & $3.00_{-0.31}^{+0.37}$ & $2.71_{-0.27}^{+0.29}$ & $4.27_{-0.90}^{+1.29}$ \\
\hline 57,109 & 66.5 & $3.44_{-0.37}^{+0.56}$ & $2.23_{-0.25}^{+0.21}$ & $4.96_{-1.19}^{+2.30}$ \\
\hline 57,168 & 121.1 & $2.86_{-1.07}^{+1.98}$ & $1.89_{-0.82}^{+1.96}$ & $1.72_{-0.60}^{+2.86}$ \\
\hline 57,177 & 129.5 & $3.22_{-1.37}^{+2.06}$ & $1.55_{-0.62}^{+1.96}$ & $1.84_{-0.80}^{+3.07}$ \\
\hline 57,185 & 136.9 & $3.57_{-1.42}^{+1.93}$ & $1.46_{-0.50}^{+1.39}$ & $2.46_{-1.21}^{+3.69}$ \\
\hline
\end{tabular}

Note.

${ }^{\mathrm{a}}$ Fits to the optical and UV SED, giving the temperature $\left(T_{\mathrm{BB}}\right)$, radius $\left(R_{\mathrm{BB}}\right)$, and luminosity $\left(L_{\mathrm{BB}}\right)$. Epochs marked with an asterisk were estimated from $r$-band images only (see Section 3).

$R_{\mathrm{BB}} \approx 1 \times 10^{14} \mathrm{~cm}$ during the rise, up to a maximum of $3 \times 10^{14} \mathrm{~cm}\left(4300 R_{\odot}\right)$ during peak, placing the emission at $\sim 23$ tidal radii from the SMBH. This value is similar to those reported for other optical TDEs (Hung et al. 2017; Wevers et al. 2017).

To further constrain the characteristics of iPTF15af, we used the same Swift $U V W 2, U V M 2$, and $U V W 1$ bands, together with the difference-imaging $g$ and $r$ photometry, to model the light curve with the open-source code MOSFiT (Guillochon et al. 2018), based on the TDE model of Guillochon \& Ramirez-Ruiz (2013). In order to avoid host galaxy contamination, we excluded the bands $U, B$, and $V$. Provided that the mass of the SMBH was available from the literature (see Section 2.1), 


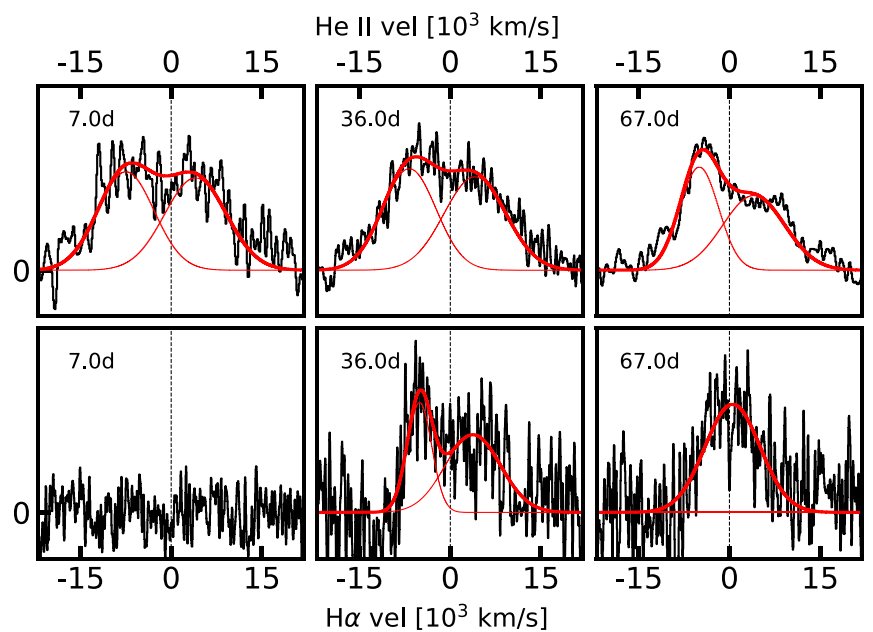

Figure 8. Best-fit line profiles to the residual emission component in iPTF15af for $\mathrm{He}$ II $\lambda 4686$ (top) and $\mathrm{H} \alpha$ (bottom). The center of the emission lines for each element has been placed at zero velocity. The flux density has been normalized for visualization purposes.

we used it as our prior probability, allowing our uniform distribution to vary within $3 \sigma$.

The light curves for a set of best-fit models are shown in Figure 7. The parameters derived from the simulation correspond to a tidal disruption of a star with mass $M_{\text {star }}=$ $2.6_{-0.9}^{+1.1} M_{\odot}$ by a black hole with $\log \left(M_{\mathrm{BH}} / M_{\odot}\right)=7.7_{-0.5}^{+0.1}$, consistent within the errors with our literature prior. This mass would be on the heavy end for the sample of TDE fits from Mockler et al. (2019), similar to the one computed for PS1-10jh. The estimated disruption time of the star is at $-38.56_{-7.0}^{+9.6}$ days. The impact parameter, defined as the ratio between the star's disruption radius and the radius of the star at pericenter $R_{T} / R_{p}=\beta=0.10_{-0.1}^{+0.36}$, is the lowest compared to the previous sample. The value $\beta<1$ would imply an extremely shallow encounter, corresponding to only a partial disruption (Guillochon $\&$ Ramirez-Ruiz 2013). Since the event is among the brightest in the sample, its luminosity could be attributed to a highly efficient conversion of $\dot{M}$ to luminosity of $\epsilon=0.23_{-0.1}^{+0.1}$. The reddening in the host estimated by the model is $\log \left(n_{\mathrm{H}} / \mathrm{cm}^{-2}\right)=20.9_{-0.1}^{+0.1}$, corresponding to an extinction of $A(V) \approx 0.4 \mathrm{mag}$ when assuming the relation provided by Güver \& Özel (2009).

\section{Spectroscopic Analysis}

\subsection{Optical Spectroscopy}

The optical spectroscopic evolution of iPTF15af is shown in the left panel of Figure 3. In the last two spectra, taken at 4 and 5 months after the event, the BELs have disappeared, with the exception of a residual flux possibly associated with $\mathrm{H} \alpha$ at +121 days. However, the contribution from a blue continuum is still noticeable in both spectra, as compared with the quiescent host at +1035 days.

For our line analysis, we used the late-time (+1035 days) spectrum of the galaxy to perform host subtraction, following the method described by Blagorodnova et al. (2017). To ensure that the TDE features were completely gone, we compared our spectrum to the archival SDSS pre-flare spectrum, taken $\sim 13 \mathrm{yr}$ before the detection of the transient. Except a small scaling factor, we found no significant deviation between the two. We then fit simultaneously the host and a blackbody component to the TDE emission spectrum and subtracted this fit from the transient spectrum. A low-order polynomial was used to level up any leftover broad component in the continuum. The residuals are shown in the right panel of Figure 3.

Using the Python package lmfit, we model the emission lines of the residual spectra with one and two Gaussian profiles and a broad exponential profile for the local continuum. The best fits are shown in Figure 8, and the measurements are logged in Table 6.

The $\mathrm{H} \alpha$ line is not detected in our first residual spectrum. However, at later epochs emission is present with possibly two distinct components at +36 days, with velocities similar to those of the He II line. Toward +67 days, it evolves into a single broad $\left(\sim 11,000 \mathrm{~km} \mathrm{~s}^{-1}\right)$ line centered on its rest-frame wavelength.

The He II $\lambda 4686$ line is clearly detected in all three spectra, which show a bimodal profile. While the redshifted wing has constant $v \approx 4000 \mathrm{~km} \mathrm{~s}^{-1}$ and an FWHM of $\sim 12,000 \mathrm{~km} \mathrm{~s}^{-1}$, there is a noticeable narrowing of the blueshifted component at later times. The velocity shifts toward lower values, and the emission becomes narrower, evolving from an average FWHM of $\sim 11,000$ to $7500 \mathrm{~km} \mathrm{~s}^{-1}$.

The blueshifted component in the double-peaked He II region is very interesting. Previous spectroscopic studies of optical TDEs attributed the blueshifted component to a blend of C III/N III, usually detected in Wolf-Rayet stars and supernovae having high temperatures (Gezari et al. 2015; Brown et al. 2018). Figure 9 shows that the centroid of this line appears to be blueshifted with respect to the rest-frame wavelengths of $\mathrm{N}$ III $\lambda 4641$ and C III $\lambda 4649$. Here we propose that this line and other emission features blueward of $4500 \AA$ are caused by the Bowen fluorescence mechanism.

The Bowen fluorescence mechanism (Bowen 1934, 1935) was initially proposed to explain the unusually strong metallic lines observed in planetary nebulae. The source of the emission lies in the recombination of fully ionized He II and its deexcitation toward its ground state. While the jumps between the outer orbits produce optical emission (such as the He II $\lambda 4686$ line), the final transition corresponding to the He II Ly $\alpha$ line produces an EUV $303.780 \AA$ photon. After scattering within the nebula, this photon can either be reabsorbed by another He II atom or enter in resonance with the O III lines at $303.800 \AA$ (O1) and $303.695 \AA$ (O3). The de-excitation of these lines produces the primary and secondary Bowen decays in the optical, following a tertiary decay emitting a $374.436 \AA$ photon.

The O III line photons created here can easily escape from the nebula, resulting in transitions at 3047, 3133, 3312, 3341, 3444, and $3760 \AA$. However, the EUV photons are likely to be reabsorbed, this time by the resonance $\mathrm{N}$ III line, with transitions at 374.434 and $374.442 \AA$. The recombination of the nitrogen atom toward its ground state is converted into a primary transition at $4640 \AA$ and a secondary one at $4100 \AA$, along with a $452 \AA$ photon. While the EUV photons are optically thick and suffer multiple absorptions and re-emissions within the nebula, the optical photons will easily escape. Observations of planetary nebulae suggest that high optical depths are required for the Bowen fluorescence to work (Selvelli et al. 2007), as the O III photons would need to suffer several scatterings in order to increase the pumping efficiency for N III.

Bowen fluorescence was predicted to occur also around accreting black holes several decades ago (Netzer et al. 1985). However, only recently has it been robustly identified in 


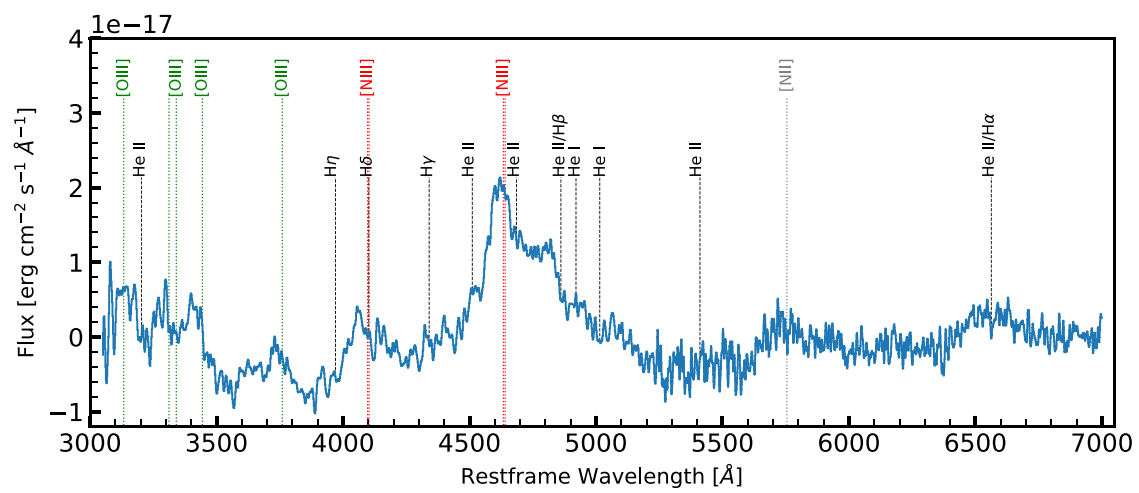

Figure 9. Identification of Bowen fluorescence lines in the spectrum at +67 days. The [N III] lines are marked in red and [O III] lines in green. The centroids of these forbidden lines appear shifted by $-3000 \mathrm{~km} \mathrm{~s}^{-1}$.

Table 6

Fit Parameters for the Optical Broad Lines

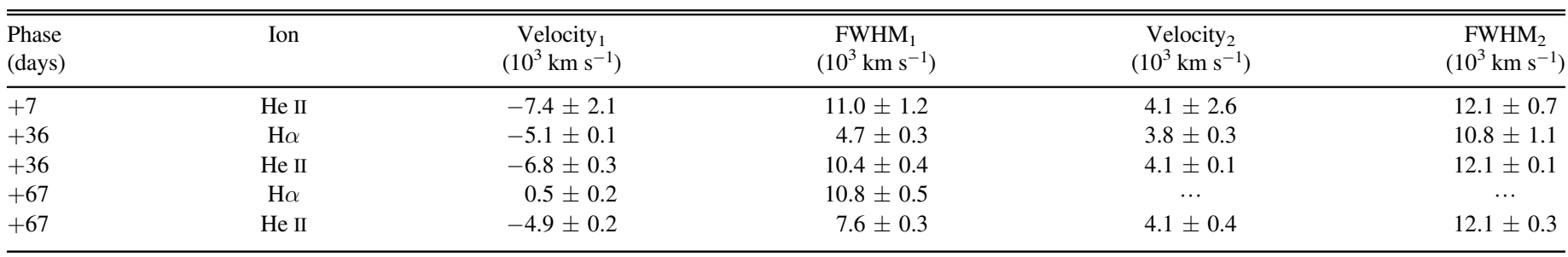

Note.

a The index "1" indicates the blueshifted component and the index " 2 " the redshifted one.

Table 7

Narrow Absorption in UV Spectra of iPTF15af

\begin{tabular}{|c|c|c|c|c|c|c|}
\hline Ion & $\begin{array}{l}\lambda_{0} \\
(\AA)\end{array}$ & $z$ & $\begin{array}{l}\lambda_{\text {obs }} \\
(\AA)\end{array}$ & $\begin{array}{c}\text { FWHM } \\
(\AA)\end{array}$ & $\begin{array}{l}\text { EW } \\
(\AA)\end{array}$ & $\begin{array}{l}\text { Velocity } \\
\left(\mathrm{km} \mathrm{s}^{-1}\right)\end{array}$ \\
\hline N I & 1200.0 & 0.0 & $1200.3 \pm 0.2$ & 2.4 & 0.32 & $75 \pm 50$ \\
\hline $\operatorname{Ly} \alpha$ & 1215.9 & 0.0 & $1214.68 \pm 0.3$ & $\cdots$ & $\ldots$ & $-300 \pm 74$ \\
\hline Si II & 1260.4 & 0.0 & $1260.06 \pm 0.1$ & 2.6 & 0.7 & $-81 \pm 24$ \\
\hline $\mathrm{BL}$ & 1302.0 & 0.0 & $1303.0 \pm 0.3$ & 2.4 & 0.2 & $230 \pm 70$ \\
\hline $\mathrm{C}$ II & 1334.5 & 0.0 & $1335.0 \pm 0.1$ & 3.21 & 0.90 & $112 \pm 22$ \\
\hline Si IV & 1393.8 & 0.0 & $1394.2 \pm 0.1$ & 3.86 & 0.54 & $86 \pm 22$ \\
\hline C IV & 1548.2 & 0.0 & $1548.5 \pm 0.3$ & 1.13 & 0.38 & $58 \pm 58$ \\
\hline Fe II & 2600.2 & 0.0 & $2602.0 \pm 0.3$ & 4.7 & 1.20 & $208 \pm 35$ \\
\hline $\mathrm{Mg}$ II & 2803.5 & 0.0 & $2801.1 \pm 0.3$ & $\cdots$ & $\cdots$ & $-257 \pm 32$ \\
\hline $\operatorname{Ly} \alpha$ & 1215.7 & 0.07897 & $1311.6 \pm 0.2$ & 2.65 & 0.96 & $-100 \pm 50$ \\
\hline C II & 1334.5 & 0.07897 & $1439.4 \pm 0.4$ & $\ldots$ & $\ldots$ & $-90 \pm 90$ \\
\hline C IV & 1548.2 & 0.07897 & $1669.7 \pm 0.6$ & $\cdots$ & $\ldots$ & $-140 \pm 110$ \\
\hline C IV & 1550.8 & 0.07897 & $1672.2 \pm 0.7$ & $\cdots$ & $\cdots$ & $-200 \pm 125$ \\
\hline
\end{tabular}

TDEs (present work) and AGN flares (Trakhtenbrot et al. 2019).

Figure 9 shows the main Bowen transition lines overplotted on the spectrum taken at +67 days. In the earlier spectra at +7 and +36 days we can also identify the lines associated with nitrogen $\lambda \lambda 4100,4640$. However, the broader line profile at those epochs makes the identification of the oxygen lines challenging.

In addition, in the early-time spectra we tentatively identify an emerging line around $5700 \AA$, close to the He I $\lambda 5875$ region. However, the origin of this line is less clear, as its blueshift velocity would be around $-9000 \mathrm{~km} \mathrm{~s}^{-1}$, higher than the one observed for $\mathrm{He}$ II and $\mathrm{H} \alpha\left(-7500\right.$ to $\left.-5000 \mathrm{~km} \mathrm{~s}^{-1}\right)$. Provided that other nitrogen lines were detected, another possible candidate would be the N II forbidden line, with a wavelength of $5754.8 \AA$.

\subsection{UV Spectroscopy}

The UV spectrum of iPTF15af, corrected for Milky Way extinction, is shown in Figure 4. Assuming negligible UV flux contribution from the host galaxy, we use the featureless part of the spectrum with $\lambda \geqslant 2000 \AA$ to fit a blackbody spectrum. Our best fit provides a temperature of $43,300_{-1,500}^{+1,700} \mathrm{~K}$, consistent with the value derived from our Swift SED analysis at a similar epoch, as shown in Figure 6. 


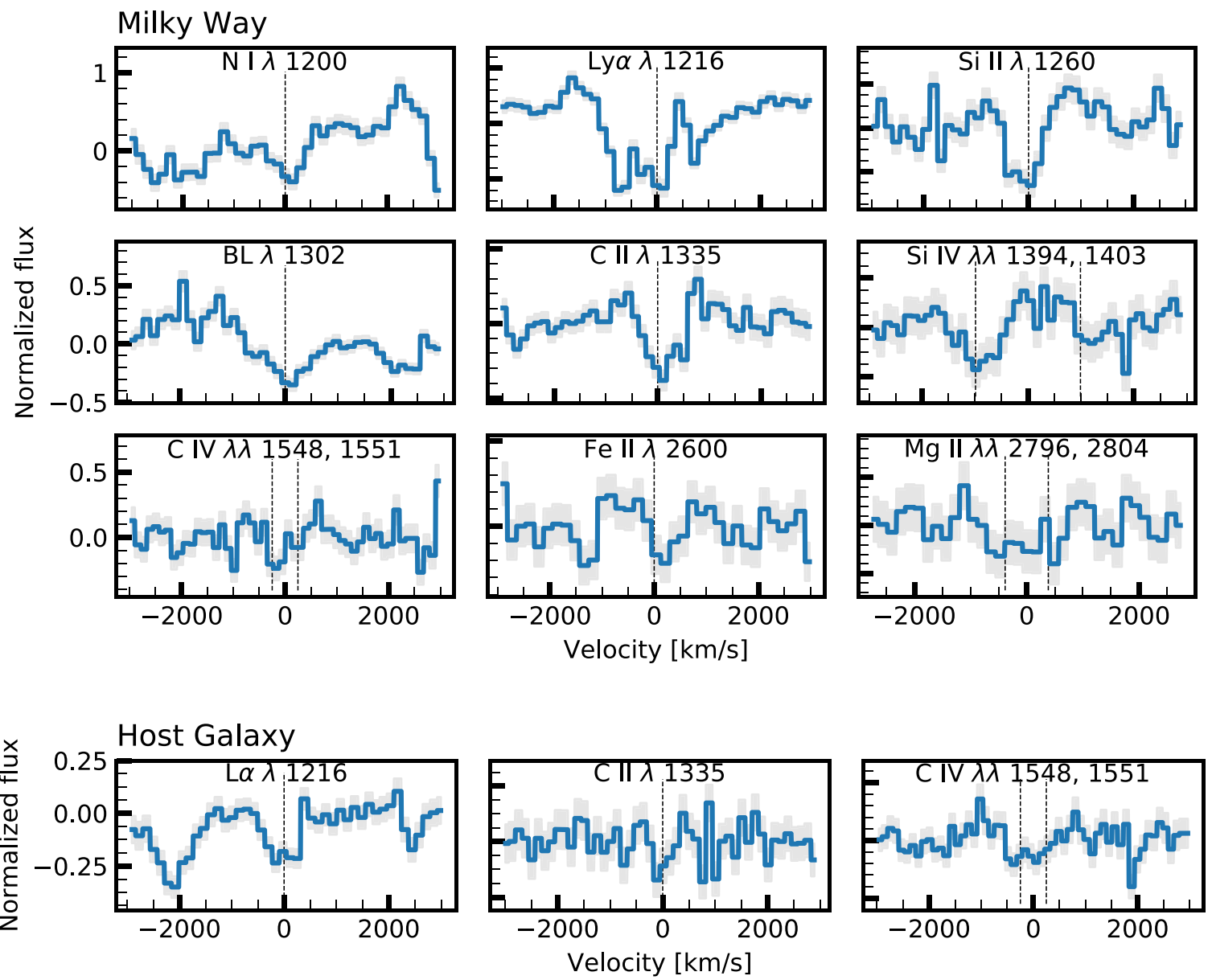

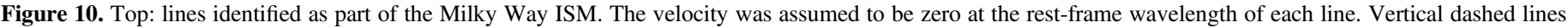
show the velocity of each line. The gray shaded area shows the spectrum $1 \sigma$ uncertainty. Bottom: lines identified at the redshift of the host galaxy.

The spectrum exhibits a combination of narrow absorption lines of low-ionization elements both in the Milky Way and in the TDE host galaxy and broad lines associated with highly ionized outflows in the host. The description of each set of features is detailed below and the measurements are provided in Table 7.

\subsubsection{Milky Way Absorption}

Figure 10 shows the line identification for elements close to their rest-frame values. Although the uncertainties in the flux appear to be small, several lines show signs of blending, biasing the line-center measurements. The average resolution of our FUV $\left(\sim 300 \mathrm{~km} \mathrm{~s}^{-1}\right)$ and NUV $\left(\sim 400 \mathrm{~km} \mathrm{~s}^{-1}\right)$ spectra makes the precise identification of nearby absorption lines challenging. Tentatively, we identified several transitions associated with the Galactic interstellar medium (ISM), containing low-ionization elements $\mathrm{NI}(14.5 \mathrm{eV})$, Ly $\alpha$ (13.6 eV), Si II $(16.3 \mathrm{eV}), \mathrm{C}$ II $(24.4 \mathrm{eV})$, Fe II $(16.2 \mathrm{eV})$, and possibly $\mathrm{Mg}$ II $(15.0 \mathrm{eV})$. We identify and mark in the spectrum in Figure 4 the geocoronal airglow lines of Ly $\alpha$, O I $\lambda 1304$, $\mathrm{O}$ I] $\lambda 1356$, and [O II] $\lambda 2471$. The high-ionization metal states correspond to SiIV $(45.1 \mathrm{eV})$ and CIV $(64.5 \mathrm{eV})$. Around $1302 \AA$ we identify an absorption corresponding to a blend of O I $\lambda 1302$ and Si II $\lambda 1304$ lines (identified as BL).

\subsubsection{Host Galaxy Absorption}

In agreement with the UV spectroscopic signature for ASASSN-14li, the spectrum of iPTF15af also shows weak
Ly $\alpha$ absorption from the host. The majority of low-ionization elements are also missing. Some narrow absorption lines that were identified correspond to Ly $\alpha$ at $1216 \AA$, C II $\lambda 1334$, and a higher-ionization line corresponding to the C IV $\lambda \lambda 1548,1551$ doublet $(64.5 \mathrm{eV})$. The $\mathrm{N} V \lambda \lambda 1239,1243$ doublet, if present, is likely blended into the $\mathrm{C}$ II line at $1335 \AA$ at Galactic redshift. We also measure a slight blueshift in our lines, with velocities of $90-200 \mathrm{~km} \mathrm{~s}^{-1}$. These values would be consistent with the motion of gas inside the host galaxy. However, there is a chance of a low-velocity outflow in this TDE as well, similar to the one observed for ASASSN-14li (Miller et al. 2015).

\subsubsection{Broad Lines}

According to the classical definition by Weymann et al. (1981), a BAL is a trough at $\geqslant 10 \%$ below the continuum level, which extends for more than $2000 \mathrm{~km} \mathrm{~s}^{-1}$. This definition has been formalized by the so-called "balnicity index" (Weymann et al. 1991), computing the equivalent width of the broad $\left(\geqslant 2000 \mathrm{~km} \mathrm{~s}^{-1}\right)$ absorption line troughs for each element. Using the best-fit blackbody spectrum as our continuum level, we compute that iPTF15af has balnicity indices of 3856 for Si V, 3495 for NV, and 3392 for C IV, consistent with the distribution observed for BAL QSOs (Gibson et al. 2009). The main BALs in the spectrum of iPTF15af have been identified and marked in Figure 4.

We also identified a feature in the region around $1100 \AA$, known to contain the resonance multiplet of Fe III (UV1), with 
Table 8

Fit Parameters for the UV Broad Lines ${ }^{\mathrm{a}}$

\begin{tabular}{lcccc}
\hline \hline Ion & $\begin{array}{c}\text { Velocity } \\
\left(10^{3} \mathrm{~km} \mathrm{~s}^{-1}\right)\end{array}$ & $\begin{array}{c}\text { FWHM }_{\text {abs }} \\
\left(10^{3} \mathrm{~km} \mathrm{~s}^{-1}\right)\end{array}$ & $\begin{array}{c}\text { Velocity }_{\text {em }} \\
\left(10^{3} \mathrm{~km} \mathrm{~s}^{-1}\right)\end{array}$ & $\begin{array}{c}\mathrm{FWHM}_{\text {em }} \\
\left(10^{3} \mathrm{~km} \mathrm{~s}^{-1}\right)\end{array}$ \\
\hline Si IV & $-6.1 \pm 0.5$ & $11.0 \pm 2.2$ & $\cdots$ & $\ldots$ \\
N III] & $\cdots$ & $\cdots$ & $-0.2 \pm 0.3$ & $10.8 \pm 1.0$ \\
He II & $\ldots$ & $\ldots$ & $2.6 \pm 0.4$ & $4.1 \pm 0.9$ \\
C IV & $-5.3 \pm 0.5$ & $9.1 \pm 1.4$ & $-1.6 \pm 6.2$ & $14.0 \pm 4.0$ \\
P V & $-4.60 \pm 0.2$ & $7.4 \pm 0.8$ & $\cdots$ & $\cdots$ \\
N V & $-5.1 \pm 0.2$ & $7.7 \pm 0.5$ & $4.2 \pm 0.1$ & $6.1 \pm 0.5$ \\
\hline
\end{tabular}

Note.

${ }^{a}$ Identified in iPTF15af. The suffix em symbolizes the values corresponding to the emission, and abs those corresponding to the absorption components. The elements are ordered from lower to higher ionization potentials: Si IV$45.1 \mathrm{eV} ; \mathrm{N} \mathrm{IIII} \longrightarrow 47.5 \mathrm{eV}$; He II—54.4 eV; C IV—64.5 eV; P V—65.0 eV; N V $-97.9 \mathrm{eV}$.

a rest wavelength of $1122.5 \AA$. However, based on the similarity between the spectra of iPTF15af and high-ionization BAL QSOs (HiBAL), we attribute the broad depression in that region to $\mathrm{P} V \lambda \lambda 1118,1128(65.0 \mathrm{eV})$, observed in $3.0 \%-6.2 \%$ of BAL QSOs (Hamann 1998; Capellupo et al. 2017).

Figure 4 shows several BELs that were also identified at the host redshift. We detect strong emission from $\mathrm{N} \mathrm{III]} \lambda 1759$ $(47.5 \mathrm{eV})$ and also He II $\lambda 1641(54.4 \mathrm{eV})$. The resonance line transitions produced by C IV $\lambda \lambda 1548,1551(64.5 \mathrm{eV}), \mathrm{Si}$ IV $\lambda \lambda 1394,1403(45.1 \mathrm{eV})$, and $\mathrm{N} \mathrm{V} \lambda \lambda 1239,1243(97.9 \mathrm{eV})$ are also present, accompanied by broad blueshifted absorption analogous to BAL QSOs.

Similar to other TDEs in quiescent galaxies (Cenko et al. 2016; Brown et al. 2018), we notice the lack of emission for the low-ionization lines of $\mathrm{Mg}$ II $\lambda \lambda 2796,2804$, Al III $\lambda \lambda 1854$, 1862, and Fe II. Furthermore, C III] $\lambda 1908$, having a similar ionization potential to that of $\mathrm{N} \mathrm{III]}(47.9 \mathrm{eV}$ vs. $47.5 \mathrm{eV})$, is also absent. Since the ratio $\mathrm{C} \mathrm{III]/N} \mathrm{III]} \mathrm{has} \mathrm{only} \mathrm{a} \mathrm{moderate}$ dependence on the physical conditions of the gas (under local thermodynamic equilibrium conditions), the lack of $\mathrm{C}$ in the spectra of TDEs has been attributed to the unusually high abundance ratio of $\mathrm{N}$ to $\mathrm{C}$ in the debris of the disrupted star (Kochanek 2016; Yang et al. 2017).

Table 8 shows the bulk velocity and the FWHM of each broad line, as modeled with combined absorption and emission Gaussian line profiles. He II and N III] are only detected in emission, with their centroids shifted by $\sim+2600$ and $-200 \mathrm{~km} \mathrm{~s}^{-1}$, respectively. The observed widths of the emission lines are in the $10^{4} \mathrm{~km} \mathrm{~s}^{-1}$ regime, corresponding to a virialized gas orbiting a $\log \left(M_{\mathrm{BH}} / M_{\odot}\right)=6.88 \mathrm{SMBH}$ at a distance of $\sim 1 \times 10^{15} \mathrm{~cm}$. This value is four times larger than our inferred blackbody photospheric radius, suggesting that the line formation region is located likely in an outflow outside the continuum emitting zone.

In Figure 11, we compare the normalized absorption profile of C IV to the other high-ionization broad lines. The velocity shifts for broad absorption components in iPTF15af are $\sim-5000 \mathrm{~km} \mathrm{~s}^{-1}$, consistent with the outflow velocities of $5000-10,000 \mathrm{~km} \mathrm{~s}^{-1}$ observed in BAL QSOs. Despite some differences in the level of continuum, the widths of the absorption troughs are consistent with each other, suggesting that the absorption is produced by the same cloud.

Assuming that the outflow in iPTF15af was initiated around the bolometric peak light (as observed in the spectral signature of

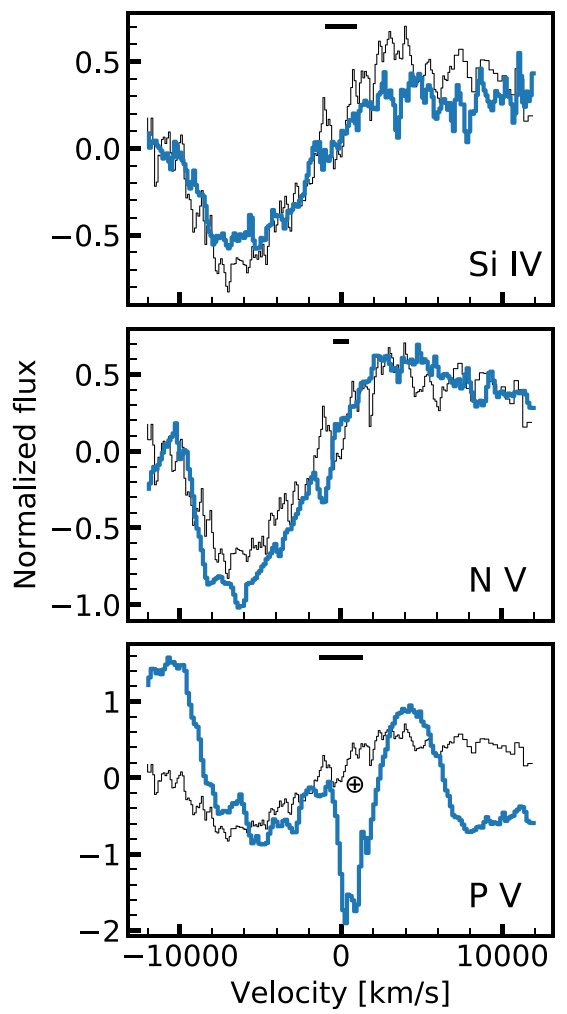

Figure 11. Comparison of the BAL of Si IV $\lambda \lambda 1394,1403, \mathrm{~N}$ V, $\lambda \lambda 1239$, 1243, and $\mathrm{P} V \lambda \lambda 1118,1128$ lines with the $\mathrm{C}$ IV $\lambda \lambda 1548,1551$ lines. The black line on the top indicates the separation in velocity of the doublet components. In the P V panel, the symbol $\oplus$ indicates absorption lines associated with the Milky Way.

PS1-11af), we can estimate the distance traveled by the cloud until the moment the UV spectrum was taken ( $\sim 30$ days). Considering the outflow's fastest absorption component traveling at $\sim 8000 \mathrm{~km} \mathrm{~s}^{-1}$, we can deduce an upper limit for the distance of the absorber of $\sim 2 \times 10^{15} \mathrm{~cm}$, which is in agreement with the virial radius computed from the emission-line width.

\section{Discussion}

iPTF15af is one of the four optical TDEs spectroscopically observed in the NUV and FUV. From these, PS16dtm and ASASSN-14li were discovered in galaxies with low-ionization nuclear emission-line regions. The latter was previously detected in X-ray and radio. Although the host of iPTF16fnl did not show any signs of activity, this event was the faintest and fastest TDE among all the optical samples explored so far. In this context, iPTF15af is quite representative of the general TDE population that we have seen so far: slowly evolving events with peak luminosities around $10^{44} \mathrm{erg} \mathrm{s}^{-1}$, generally in nonactive galaxies. iPTF15af also had nondetections in radio and had weak (if any) soft X-ray emission. The characteristics observed for this transient may be relevant for the interpretation of the bulk of TDEs observed at UV wavelengths.

A comparison of iPTF15af with other optically discovered TDEs is shown in Figure 12. Several AGN spectra are also included: a BAL QSO from Hamann (1998), a composite QSO from the SDSS spectroscopic survey (Vanden Berk et al. 2001), and the nitrogen-rich QSO J164148.19+223225.2 (Batra \& Baldwin 2014). 


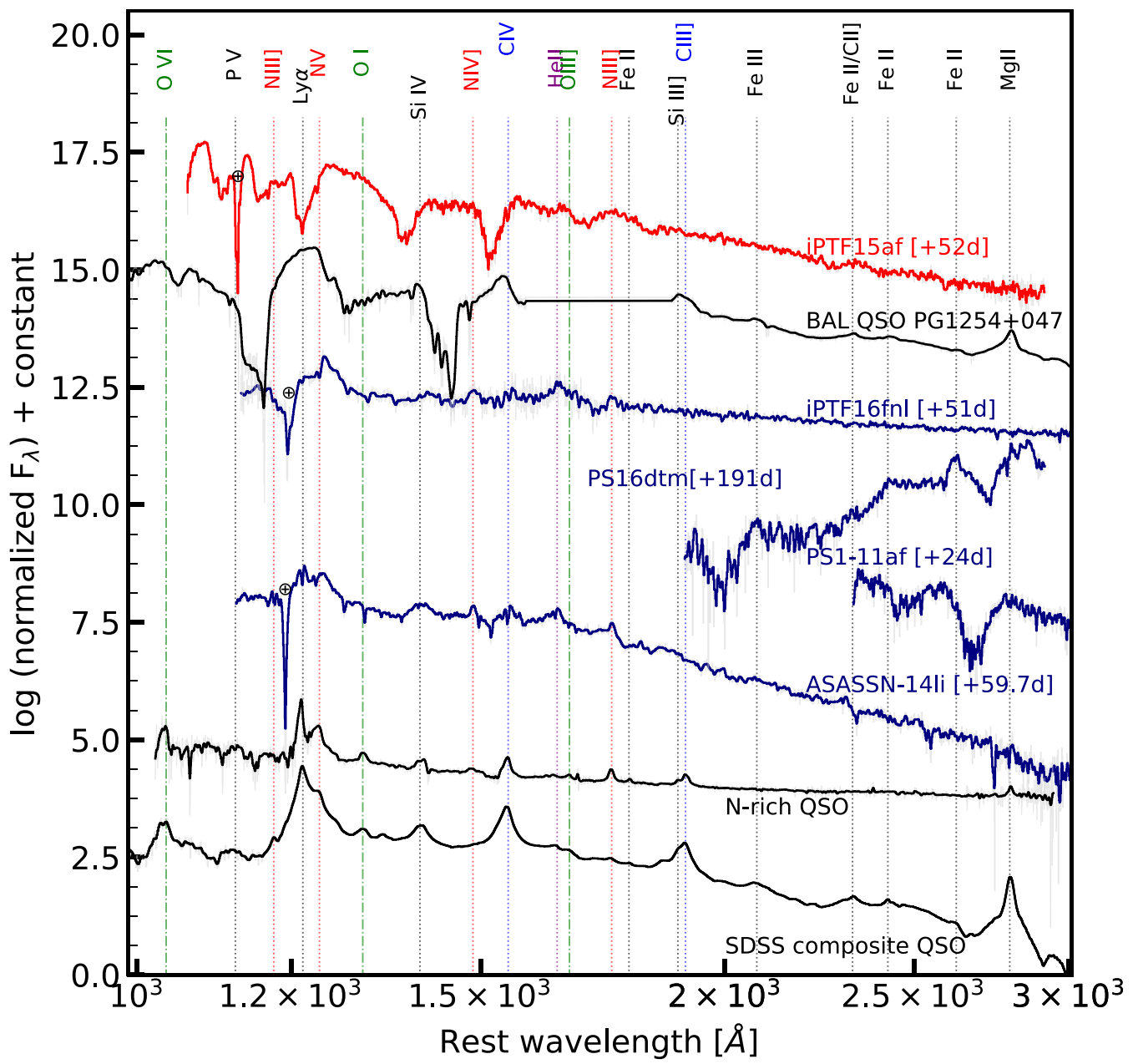

Figure 12. Comparison of HST/STIS spectrum of iPTF15af (red) with UV spectra of other TDEs (blue) and QSOs (black). From top to bottom the comparison spectra are the BAL QSO PG 1254+047 (Hamann 1998; combined HST Faint Object Spectrograph [FOS] and SDSS spectrum), TDE iPTF16fnl (Brown et al. 2018), TDE PS1-11af (Chornock et al. 2014), TDE PS16dtm (Blanchard et al. 2017), TDE ASASSN-14li (Cenko et al. 2016), the N-rich QSO spectrum of SDSS J164148.19 +223225.2 (Batra \& Baldwin 2014), and the SDSS composite QSO spectrum (Vanden Berk et al. 2001). The phase after discovery or peak for each TDE is shown in brackets. The spectra have been corrected for Galactic extinction. The $\oplus$ symbol shows the region with strong Galactic Ly $\alpha$ absorption. The most important lines identified in the spectra are marked at the top.

An initial remarkable difference is that none of the early-time emission in TDEs shows the presence of low-ionization elements such as $\mathrm{Mg}$ II $(15.0 \mathrm{eV})$ and Fe II $(16.2 \mathrm{eV})$, which are commonly observed in AGN spectra. The origin of these lines is believed to be in the partly neutral, low-ionization regions in optically thick gas clouds, which are being irradiated by X-ray photons from the central engine. For such lines to exist, the broad-line region (BLR) clouds would require a lower limit on the column density on the order of $10^{22} \mathrm{~cm}^{-2}$ (Blandford et al. 1990). The lack of strong lines redward of $1900 \AA$ can be attributed to insufficient shielding from highenergy photons, which keep the ionization continuum in the cloud well above their ionization potential of $\sim 16 \mathrm{eV}$.

The absorption components for low-ionization ions $\mathrm{Mg}$ II and possibly Fe II can be observed for two TDEs in our comparison sample: PS1-11af (Chornock et al. 2014) and PS16dtm (Blanchard et al. 2017), which also differ from other TDEs in optical wavelengths. The absorption in PS1-11af was not present in the spectrum taken at -5 days, suggesting that it formed in an outflow outside of the continuum region, analogous to our interpretation of iPTF15af.
The higher redshift of iPTF15af allows us to reach shorter wavelengths, where we putatively identify broad absorption corresponding to the ion $\mathrm{PV} \lambda \lambda 1118,1128$. This element is also observed in $\sim 5 \%$ of BAL QSOs, and its presence implies the existence of large column densities of $N_{\mathrm{H}} \approx 10^{22}-10^{23} \mathrm{~cm}^{-2}$ (Capellupo et al. 2017). In most QSOs, the PV lines do not appear saturated, providing an impression of an extreme metalto-hydrogen abundance ratio for the circumnuclear gas (Hamann 1998). However, if the absorbing outflows are concentrated in small optically thick cloudlets, they would only cover a fraction of the background emission source, supplying an extra continuum component.

As most BAL QSOs are weak in X-rays (Brandt et al. 2000; Gallagher et al. 2001), we can argue that the high column density outflows in iPTF15af would also act as an absorber for the soft $\mathrm{X}$-rays, which would explain the early-time nondetections. The delayed reflection of high-energy emission in these cloudlets would possibly generate a highly enhanced $\mathrm{He}$ II $/ \mathrm{H} \alpha$ ratio, as observed in several optical TDEs (Saxton et al. 2018). However, the drop in density at late times would allow this emission to leak out, explaining the soft X-ray brightening of ASASSN-15oi reported by Gezari et al. (2017) and Holoien et al. (2018). This 
lower density would also contribute to the narrowing of the optical line profiles, as observed in most optical TDEs (Roth \& Kasen 2018).

The lack of detectable radio and X-ray emission for iPTF15af further confirms that optical TDEs are predominantly radio-quiet (van Velzen et al. 2013), which seems to contrast with the high radio-loud fraction of AGNs among the nitrogenrich population (Jiang et al. 2008).

One traditional diagnostic of the gas density is the strength of the line C III] $\lambda 1909$, which has been extensively used in quasars to derive an upper limit for the density of the line-forming gas region (Osterbrock 1970). Collisional de-excitation of this line becomes comparable to the spontaneous radiation rate at values approaching its critical density $n_{c} \approx 3 \times 10^{9} \mathrm{~cm}^{-3}$. Hence, C III] and other semiforbidden lines, such as $\mathrm{N} \mathrm{III]} \lambda 1750\left(n_{c} \approx 2 \times\right.$ $\left.10^{10} \mathrm{~cm}^{-3}\right)$, O III] $\lambda 1663\left(n_{c} \approx 4.6 \times 10^{10} \mathrm{~cm}^{-3}\right)$, and N IV] $\lambda 1486\left(n_{c} \approx 3.4 \times 10^{10} \mathrm{~cm}^{-3}\right)$, are expected to be weaker in the interior of the BLR, where the density exceeds their critical value. These lines are expected to gain importance in the external part of the BLR, where the density is lower.

In the case of iPTF15af and other TDEs, we encounter a puzzle, as the spectrum shows unequal contributions for $\mathrm{C} \mathrm{III]}$ and $\mathrm{N}$ III]. The nitrogen atom has similar ionization potential to carbon and the same ionization structure dependencies. Naively, we would expect the less dense BLR regions to form both lines at the same time, with the $\mathrm{N} \mathrm{III]/N} \mathrm{IV} \mathrm{ratio} \mathrm{enhanced} \mathrm{in} \mathrm{a} \mathrm{similar}$ way to $\mathrm{C} \mathrm{III]/C} \mathrm{IV.} \mathrm{However,} \mathrm{as} \mathrm{previously} \mathrm{discussed} \mathrm{in} \mathrm{the}$ literature (Cenko et al. 2016; Brown et al. 2018), this assumption fails for TDE spectra. While the semiforbidden $\mathrm{N}$ III] lines are clearly detected with broad velocity spread, the C III] is lacking in all three TDEs having FUV spectra. The origin for this discrepancy has been associated with the nitrogen-enhanced composition of the stellar debris for supersolar-mass stars (Kochanek 2016; Yang et al. 2017). The carbon-nitrogenoxygen cycle would have substantially altered the star's composition with enhanced nitrogen at the expense of carbon, translated into the observed line ratios. The debris of such a star would pollute the BLR, providing a possible link with nitrogenrich QSOs (Kochanek 2016).

Additionally, the existence of strong nitrogen emission around AGNs has been discussed by several recent studies. Some support the hypothesis that strong nitrogen lines imply highly supersolar metallicity in the vicinity of the SMBH (Batra \& Baldwin 2014), increased by continuous circumnuclear star formation. Others argue that the observed strong emission is most likely attributed to an exceptionally nitrogenrich composition in the BLR, rather than overall enhanced metallicity (Matsuoka et al. 2017). The origin for this N-rich material would be linked to strong winds from the young asymptotic giant branch (AGB) stellar population in the nuclear regions of the galaxy. The studies agree, though, that the N-loud AGN sample has on average lower SMBH masses as compared to the general population in the sample. This is a good indication that these galaxies are just initiating their main growth stage via high accretion episodes.

TDE discoveries have been predominantly associated with post-starburst galaxies having high stellar densities in the bulge (French et al. 2016; Law-Smith et al. 2017; Graur et al. 2018). For these galaxies, we would expect a young stellar population actively polluting the circumnuclear regions with both metalrich supernova winds and N-enhanced AGB stellar winds. Regardless of the enrichment mechanism, TDEs would primarily be hosted in galaxies with already altered chemical abundances, translating into an unusually strong nitrogen component in their UV spectra. However, an additional mechanism would need to be invoked to explain the apparent lack of $\mathrm{C} \mathrm{III]} \mathrm{and} \mathrm{strong} \mathrm{C} \mathrm{IV} \mathrm{emission,} \mathrm{suggesting} \mathrm{that} \mathrm{the}$ composition of the star indeed plays an important role in shaping the observed UV signature of TDEs.

One important difference between iPTF15af and BAL QSOs is the shape of the absorption. While the TDE shows a smooth gradient from lower to higher velocities and a sharp cut at $v_{\max } \approx 9000 \mathrm{~km} \mathrm{~s}^{-1}$, BAL QSOs generally exhibit the opposite trend: their absorption profile is detached from their emission. Their spectra show a sharp cut at lower velocities and a broader wing corresponding to higher velocity outflows. In BAL QSOs this means that the gas in our line of sight has already been radiatively accelerated to higher speeds. Closer to the emitting region, the gas will have higher column density but lower velocity, appearing as a sharp trough in the spectra next to the emission line. In the case of iPTF15af we see that most of the absorption is caused by high-velocity gas transitioning to lower-velocity outflows.

Outflows with $\sim 10^{4} \mathrm{~km} \mathrm{~s}^{-1}$ have been predicted to occur in TDEs with radiatively inefficient accretion flows (Metzger \& Stone 2016). Here we present UV spectroscopic data that confirm the existence of the outflow, and we discuss the acceleration mechanism. For a given SMBH mass, the maximum velocity of the TDE outflow will depend on the Eddington ratio of the flare, the density of the gas, and its distance to the central engine, as shown for AGNs (Risaliti \& Elvis 2010). At smaller radii, the gas will become overionized and the radiative wind will fail. At larger radii, the UV radiation field will be too weak to provide a noticeable acceleration. Therefore, the highest velocity for the bulk of stellar debris is likely to be achieved for dense gas at intermediate radius $\left(\sim 100 R_{s}\right)$. The remaining bound material returning to the SMBH will progressively feel weaker acceleration, as the central source will be quickly fading with time, but also the material will be less dense. As the density of the ejecta decreases, it will become easier for the ionizing radiation to eventually penetrate this material and escape to infinity, making the mechanism less efficient. Continuous monitoring of these absorption-line profiles can provide a new tool to better understand the outflow geometry in TDEs.

\section{Conclusions}

iPTF15af is a TDE discovered in the core of a galaxy with signs of a short recent starburst episode and high nuclear stellar density in its core. The photometric optical and UV evolution of this event is consistent with previously studied TDEs, such as PS1-10jh and ASASSN-15oi. The event has a comparably slow rise time of $\sim 60$ days and peaks at $L_{\text {peak }} \approx 1.5 \times 10^{44} \mathrm{erg} \mathrm{s}^{-1}$, with an estimated temperature of $T_{\mathrm{BB}}=(40-50) \times 10^{4} \mathrm{~K}$.

The optical spectral evolution of iPTF15af shows broad characteristic $\mathrm{He}$ II and later $\mathrm{H} \alpha$ lines superposed on a blue featureless continuum, which persists beyond 4 months past discovery. During the first 3 months, the optical lines exhibit a fast evolution in their line profile, with the $\mathrm{H} \alpha$ line appearing and becoming narrower at later times. The lines are no longer detected after $\sim 120$ days post-discovery.

Our spectroscopic analysis reveals fluorescence lines of O III and $\mathrm{N}$ III, likely related to the Bowen fluorescence mechanism observed in planetary nebulae. The fluorescence between He II 
$-\mathrm{O}$ III and $\mathrm{O}$ III-N III would explain the pumping of EUV flux into optical emission.

We find that the medium producing the absorption in iPTF15af would come from a highly ionized source shielded by high-density material, justifying the lack of low-ionization lines of $\mathrm{Mg}$ II and Fe II and the appearance of BALs for highly ionized states of $\mathrm{C}, \mathrm{N}, \mathrm{Si}$, and P. The density of the gas would act as an absorber of the flare in X-rays, irradiating it toward lower-density gas enriched with nitrogen and/or metals supplied by a young stellar population in the bulge. The composition of the gas would then tip the $\mathrm{N} / \mathrm{C}$ ratio to higher values than usually found in AGNs. In addition, the nitrogenenhanced and carbon-depleted stellar debris of an evolved star can help justify the observed lack of C III in the UV spectrum.

Contrary to BAL QSOs, the BAL profiles observed in iPTF15af suggest that the highest column density material is moving at the outflow maximum velocity. We propose that the radiation pressure generated by the TDE flare at early times can supply the acceleration mechanism for this high-density gas. Future multi-epoch observations of TDEs in the UV would help to constrain the geometry, density, and kinematics of the absorbing material, allowing us to test this hypothesis. Latetime observations in X-rays are required to verify the change in the outflow's density, allowing high energy to escape.

We thank the anonymous referee, whose comments and suggestions helped to improve the paper. We are grateful to Peter Maksym, Sterl Phinney, Eliot Quataert, Clément Bonnerot, Tiara Hung, and Sjoert Van Velzen for valuable discussions and comments on this work. We are grateful to Eric Bellm, Melissa Graham, Anna Ho, and Daniel Perley for carrying out some of the spectroscopic observations and/or reductions, and Linda Strubbe for her support with the HST proposal. We thank the HST staff for the prompt scheduling of these ToO observations, as well as the PI Neil Gehrels and the Swift ToO team for the timely execution of our observations.

This work was supported by the GROWTH project funded by the National Science Foundation (NSF) under grant AST1545949. This work is part of the research program VENI, with project no. 016.192.277, which is (partly) financed by the Netherlands Organisation for Scientific Research (NWO). Support for I.A. was provided by NASA through the Einstein Fellowship Program, grant PF6-170148.

A.H. acknowledges support by the I-Core Program of the Planning and Budgeting Committee and the Israel Science Foundation. This research was supported by a Grant from the GIF, the German-Israeli Foundation for Scientific Research and Development. A.V.F.'s group at UC Berkeley is grateful for financial assistance from the TABASGO Foundation, the Christopher R. Redlich Fund, and the Miller Institute for Basic Research in Science (UC Berkeley).

Some of the data presented herein were obtained at the W. M. Keck Observatory, which is operated as a scientific partnership among the California Institute of Technology, the University of California, and the National Aeronautics and Space Administration (NASA); the observatory was made possible by the generous financial support of the W. M. Keck Foundation. This work makes use of observations from Las Cumbres Observatory. This publication makes use of data products from the Two Micron All Sky Survey, which is a joint project of the University of Massachusetts and the Infrared Processing and Analysis Center/California Institute of
Technology, funded by NASA and the NSF. This publication makes use of data products from the Wide-field Infrared Survey Explorer, which is a joint project of the University of California, Los Angeles, and the Jet Propulsion Laboratory/ California Institute of Technology, funded by NASA. Based on observations made with the NASA Galaxy Evolution Explorer. $G A L E X$ is operated for NASA by the California Institute of Technology under NASA contract NAS5-98034.

Facilities: HST (STIS), Karl G. Jansky Very Large Array, Keck:I (LRIS), PO:1.2 m, PO:1.5 m, Swift, WISE.

Software: Astropy (The Astropy Collaboration et al. 2018), emcee (Foreman-Mackey et al. 2013), FPipe (Fremling et al. 2016), lcogtsnpipe (Valenti et al. 2016), lmfit (Newville et al. 2014), lpipe http://www.astro.caltech.edu/ dperley/programs/ lpipe.html, MOSFIT (Guillochon et al. 2018), NumPy (van der Walt et al. 2011), PTFIDE (Masci et al. 2017), SciPy (Jones et al. 2001).

\section{ORCID iDs}

N. Blagorodnova (iD https://orcid.org/0000-0003-0901-1606

S. B. Cenko (D) https://orcid.org/0000-0003-1673-970X

S. R. Kulkarni ib https://orcid.org/0000-0001-5390-8563

I. Arcavi (iD https://orcid.org/0000-0001-7090-4898

G. Duggan (iD https://orcid.org/0000-0002-9256-6735

A. V. Filippenko (iD https://orcid.org/0000-0003-3460-0103

C. Fremling (i) https://orcid.org/0000-0002-4223-103X

G. Hosseinzadeh (iD https://orcid.org/0000-0002-0832-2974

E. Karamehmetoglu (i) https://orcid.org/0000-00016209-838X

A. Levan (iD https://orcid.org/0000-0001-7821-9369

F. J. Masci (i) https://orcid.org/0000-0002-8532-9395

P. E. Nugent (iD https://orcid.org/0000-0002-3389-0586

S. Veilleux (1D https://orcid.org/0000-0002-3158-6820

\section{References}

Alam, S., Albareti, F. D., Allende Prieto, C., et al. 2015, ApJS, 219, 12 Alexander, K. D., Berger, E., Guillochon, J., Zauderer, B. A., \& Williams, P. K. G. 2016, ApJL, 819, L25

Arcavi, I., Gal-Yam, A., Sullivan, M., et al. 2014, ApJ, 793, 38

Avni, Y., \& Tananbaum, H. 1982, ApJL, 262, L17

Bade, N., Komossa, S., \& Dahlem, M. 1996, A\&A, 309, L35

Batra, N. D., \& Baldwin, J. A. 2014, MNRAS, 439, 771

Bianchi, L., Herald, J., Efremova, B., et al. 2011, Ap\&SS, 335, 161

Blagorodnova, N., Gezari, S., Hung, T., et al. 2017, ApJ, 844, 46

Blanchard, P. K., Nicholl, M., Berger, E., et al. 2017, ApJ, 843, 106

Blandford, R. D., Netzer, H., Woltjer, L., Courvoisier, T. J.-L., \& Mayor, M. (ed.) 1990, Active Galactic Nuclei 97 (Berlin: Springer)

Bloom, J. S., Giannios, D., Metzger, B. D., et al. 2011, Sci, 333, 203

Bowen, I. S. 1934, PASP, 46, 146

Bowen, I. S. 1935, ApJ, 81, 1

Brandt, W. N., Laor, A., \& Wills, B. J. 2000, ApJ, 528, 637

Breeveld, A. A., Curran, P. A., Hoversten, E. A., et al. 2010, MNRAS, 406, 1687

Brown, J. S., Kochanek, C. S., Holoien, T. W.-S., et al. 2018, MNRAS, 473, 1130

Brown, T. M., Baliber, N., Bianco, F. B., et al. 2013, PASP, 125, 1031

Bruzual, G., \& Charlot, S. 2003, MNRAS, 344, 1000

Burrows, D. N., Hill, J. E., Nousek, J. A., et al. 2005, SSRv, 120, 165

Burrows, D. N., Kennea, J. A., Ghisellini, G., et al. 2011, Natur, 476, 421

Capellupo, D. M., Hamann, F., Herbst, H., et al. 2017, MNRAS, 469, 323

Cenko, S. B., Cucchiara, A., Roth, N., et al. 2016, ApJL, 818, L32

Cenko, S. B., Krimm, H. A., Horesh, A., et al. 2012, ApJ, 753, 77

Chang, Y.-Y., van der Wel, A., da Cunha, E., \& Rix, H.-W. 2015, ApJS, 219, 8

Chornock, R., Berger, E., Gezari, S., et al. 2014, ApJ, 780, 44

Condon, J. J., Cotton, W. D., Greisen, E. W., et al. 1998, AJ, 115, 1693

Drake, A. J., Djorgovski, S. G., Mahabal, A., et al. 2009, ApJ, 696, 870 
Foreman-Mackey, D., Hogg, D. W., Lang, D., \& Goodman, J. 2013, PASP, 125,306

Fremling, C., Sollerman, J., Taddia, F., et al. 2016, A\&A, 593, A68

French, K. D., Arcavi, I., \& Zabludoff, A. 2016, ApJL, 818, L21

French, K. D., Arcavi, I., \& Zabludoff, A. 2017, ApJ, 835, 176

Gallagher, S. C., Brandt, W. N., Laor, A., et al. 2001, ApJ, 546, 795

Gehrels, N., Chincarini, G., Giommi, P., et al. 2004, ApJ, 611, 1005

Generozov, A., Mimica, P., Metzger, B. D., et al. 2017, MNRAS, 464, 2481

Gezari, S., Basa, S., Martin, D. C., et al. 2008, ApJ, 676, 944

Gezari, S., Cenko, S. B., \& Arcavi, I. 2017, ApJL, 851, L47

Gezari, S., Chornock, R., Lawrence, A., et al. 2015, ApJL, 815, L5

Gezari, S., Chornock, R., Rest, A., et al. 2012, Natur, 485, 217

Gezari, S., Martin, D. C., Milliard, B., et al. 2006, ApJL, 653, L25

Gibson, R. R., Jiang, L., Brandt, W. N., et al. 2009, ApJ, 692, 758

Graur, O., French, K. D., Zahid, H. J., et al. 2018, ApJ, 853, 39

Guillochon, J., Nicholl, M., Villar, V. A., et al. 2018, ApJS, 236, 6

Guillochon, J., \& Ramirez-Ruiz, E. 2013, ApJ, 767, 25

Güver, T., \& Özel, F. 2009, MNRAS, 400, 2050

Hamann, F. 1998, ApJ, 500, 798

Henden, A. A., Templeton, M., Terrell, D., et al. 2016, yCat, 2336

Holoien, T. W.-S., Brown, J. S., Auchettl, K., et al. 2018, MNRAS, 480, 5689

Holoien, T. W.-S., Kochanek, C. S., Prieto, J. L., et al. 2016a, MNRAS, 455, 2918

Holoien, T. W.-S., Kochanek, C. S., Prieto, J. L., et al. 2016b, MNRAS, 463,3813

Hung, T., Gezari, S., Blagorodnova, N., et al. 2017, ApJ, 842, 29

Jiang, L., Fan, X., \& Vestergaard, M. 2008, ApJ, 679, 962

Jiang, N., Wang, T., Yan, L., et al. 2017, ApJ, 850, 63

Jones, E., Oliphant, T., Peterson, P., et al. 2001, SciPy: Open Source Scientific Tools for Python, http://www.scipy.org/

Just, D. W., Brandt, W. N., Shemmer, O., et al. 2007, ApJ, 665, 1004

Kochanek, C. S. 2016, MNRAS, 458, 127

Komossa, S., \& Bade, N. 1999, A\&A, 343, 775

Kormendy, J., \& Richstone, D. 1995, ARA\&A, 33, 581

Kraft, R. P., Burrows, D. N., \& Nousek, J. A. 1991, ApJ, 374, 344

Laher, R. R., Surace, J., Grillmair, C. J., et al. 2014, PASP, 126, 674

LaMassa, S. M., Cales, S., Moran, E. C., et al. 2015, ApJ, 800, 144

Law-Smith, J., Ramirez-Ruiz, E., Ellison, S. L., \& Foley, R. J. 2017, ApJ, 850,22

Mainzer, A., Bauer, J., Cutri, R. M., et al. 2014, ApJ, 792, 30

Masci, F. J., Laher, R. R., Rebbapragada, U. D., et al. 2017, PASP, 129, 014002

Matsuoka, K., Nagao, T., Maiolino, R., et al. 2017, A\&A, 608, A90

Mendel, J. T., Simard, L., Palmer, M., Ellison, S. L., \& Patton, D. R. 2014, ApJS, 210, 3

Metzger, B. D., \& Stone, N. C. 2016, MNRAS, 461, 948

Miller, J. M., Kaastra, J. S., Miller, M. C., et al. 2015, Natur, 526, 542

Mockler, B., Guillochon, J., \& Ramirez-Ruiz, E. 2019, ApJ, 872, 151
Netzer, H., Elitzur, M., \& Ferland, G. J. 1985, ApJ, 299, 752

Newville, M., Stensitzki, T., Allen, D. B., \& Ingargiola, A. 2014, LMFIT: Nonlinear Least-Square Minimization and Curve-Fitting for Python, Zenodo, doi: $10.5281 /$ zenodo. 11813

Ofek, E. O., Laher, R., Surace, J., et al. 2012, PASP, 124, 854

Oke, J. B., Cohen, J. G., Carr, M., et al. 1995, PASP, 107, 375

Oke, J. B., \& Gunn, J. E. 1983, ApJ, 266, 713

Osterbrock, D. E. 1970, ApJ, 160, 25

Poole, T. S., Breeveld, A. A., Page, M. J., et al. 2008, MNRAS, 383, 627

Rau, A., Kulkarni, S. R., Law, N. M., et al. 2009, PASP, 121, 1334

Rees, M. J. 1988, Natur, 333, 523

Risaliti, G., \& Elvis, M. 2010, A\&A, 516, A89

Roming, P. W. A., Kennedy, T. E., Mason, K. O., et al. 2005, SSRv, 120, 95

Roth, N., \& Kasen, D. 2018, ApJ, 855, 54

Saxton, C. J., Perets, H. B., \& Baskin, A. 2018, MNRAS, 474, 3307

Saxton, R. D., Read, A. M., Esquej, P., et al. 2012, A\&A, 541, A106

Schlafly, E. F., \& Finkbeiner, D. P. 2011, ApJ, 737, 103

SDSS Collaboration, Albareti, F. D., Allende Prieto, C., et al. 2017, ApJS, 233, 25

Selvelli, P., Danziger, J., \& Bonifacio, P. 2007, A\&A, 464, 715

Simard, L., Mendel, J. T., Patton, D. R., Ellison, S. L., \& McConnachie, A. W. 2011, ApJS, 196, 11

Skrutskie, M. F., Cutri, R. M., Stiening, R., et al. 2006, AJ, 131, 1163

Stern, D., Assef, R. J., Benford, D. J., et al. 2012, ApJ, 753, 30

Stone, N. C., Kesden, M., Cheng, R. M., \& van Velzen, S. 2019, GreGr, 51, 30

The Astropy Collaboration, Price-Whelan, A. M., Sipőcz, B. M., et al. 2018 , AJ, 156, 123

Trakhtenbrot, B., Arcavi, I., Ricci, C., et al. 2019, NatAs, in press (doi:10. 1038/s41550-018-0661-3)

Valenti, S., Howell, D. A., Stritzinger, M. D., et al. 2016, MNRAS, 459, 3939

van der Walt, S., Colbert, S. C., \& Varoquaux, G. 2011, CSE, 13, 22

van Velzen, S., Anderson, G. E., Stone, N. C., et al. 2016a, Sci, 351, 62

van Velzen, S., Farrar, G. R., Gezari, S., et al. 2011, ApJ, 741, 73

van Velzen, S., Frail, D. A., Körding, E., \& Falcke, H. 2013, A\&A, 552, A5

van Velzen, S., Mendez, A. J., Krolik, J. H., \& Gorjian, V. 2016b, ApJ, 829, 19

van Velzen, S., Stone, N. C., Metzger, B. D., et al. 2018, ApJ, submitted (arXiv:1809.00003)

Vanden Berk, D. E., Richards, G. T., Bauer, A., et al. 2001, AJ, 122, 549

Voges, W., Aschenbach, B., Boller, T., et al. 1999, A\&A, 349, 389

Wevers, T., van Velzen, S., Jonker, P. G., et al. 2017, MNRAS, 471, 1694

Weymann, R. J., Carswell, R. F., \& Smith, M. G. 1981, ARA\&A, 19, 41

Weymann, R. J., Morris, S. L., Foltz, C. B., \& Hewett, P. C. 1991, ApJ, 373,23

Willingale, R., Starling, R. L. C., Beardmore, A. P., Tanvir, N. R., \& O’Brien, P. T 2013, MNRAS, 431, 394

Wright, E. L., Eisenhardt, P. R. M., Mainzer, A. K., et al. 2010, AJ, 140, 1868

Yang, C., Wang, T., Ferland, G. J., et al. 2017, ApJ, 846, 150

Yaron, O., \& Gal-Yam, A. 2012, PASP, 124, 668 